\title{
Dynamic imperfections and optimized feedback design in the Compact Linear Collider main linac
}

\author{
Peder Eliasson* \\ European Organization for Nuclear Research (CERN), CH-1211 Geneva 23, Switzerland, \\ and Department of Physics and Astronomy, Uppsala University, Box 516, SE-75120 Uppsala, Sweden
}

(Received 11 February 2008; published 14 May 2008)

The Compact Linear Collider (CLIC) main linac is sensitive to dynamic imperfections such as element jitter, injected beam jitter, and ground motion. These effects cause emittance growth that, in case of ground motion, has to be counteracted by a trajectory feedback system. The feedback system itself will, due to jitter effects and imperfect beam position monitors (BPMs), indirectly cause emittance growth. Fast and accurate simulations of both the direct and indirect effects are desirable, but due to the many elements of the CLIC main linac, simulations may become very time consuming. In this paper, an efficient way of simulating linear (or nearly linear) dynamic effects is described. The method is also shown to facilitate the analytic determination of emittance growth caused by the different dynamic imperfections while using a trajectory feedback system. Emittance growth expressions are derived for quadrupole, accelerating structure, and beam jitter, for ground motion, and for noise in the feedback BPMs. Finally, it is shown how the method can be used to design a feedback system that is optimized for the optics of the machine and the ground motion spectrum of the particular site. This feedback system gives an emittance growth rate that is approximately 10 times lower than that of traditional trajectory feedbacks. The robustness of the optimized feedback system is studied for a number of additional imperfections, e.g., dipole corrector imperfections and faulty knowledge about the machine optics, with promising results.

DOI: 10.1103/PhysRevSTAB.11.051003

PACS numbers: $29.20 .-\mathrm{c}$

\section{INTRODUCTION}

The performance of the future Compact Linear Collider (CLIC) [1] relies heavily on efficient preservation of emittance throughout the machine. Both static and dynamic imperfections influence the emittance and cause severe emittance growth if not mitigated.

Beam-based alignment and so-called emittance tuning bumps [2,3] have been proved an efficient means for correction of static imperfections. Dynamic imperfections, on the other hand, can be partly corrected with various feedback techniques. In case of the CLIC main linac, which due to its many elements is sensitive to imperfections, trajectory feedbacks are foreseen to be used to reduce emittance growth $[4,5]$. By using beam position monitor (BPM) readings, the trajectory feedbacks resteer the beam locally to the ideal trajectory. Without such feedbacks, dynamic misalignments would cause increasing deviations from the ideal trajectory and the emittance would quickly become unacceptable.

The dynamic imperfection with the strongest influence on emittance is ground motion, which in this paper will be described using the simple $A T L$ model [6]. Because of the movements of the ground, elements slowly drift away from their ideal positions, leading to an imperfect beam trajectory. The increasing element displacements cause the emittance to grow with time. If the movements are not too fast, the emittance may be efficiently preserved by using trajectory feedbacks to correct the beam trajectory. Since the

\footnotetext{
*peder.eliasson@gmail.com
}

BPMs used for feedback have a finite resolution, the feedback system will itself give rise to some emittance growth. This effect is also important to consider.

In addition to the slow drifts, which may be corrected by feedback, an important type of dynamic imperfections is so-called jitter. Here, jitter indicates a nondrifting variation, in, for example, element position, that is rapid enough to appear independent from one pulse to the next. Because of the short pulse duration in CLIC, the trajectory feedbacks cannot read out the BPMs and then apply the desired global correction (using correctors along the whole linac) to the same pulse. The correction will instead be applied to the following pulse, and, consequently, jitter cannot be corrected using this feedback system. Jitter effects studied in this paper include quadrupole and accelerating structure jitter, as well as jitter in the vertical position of the injected beam. If a feedback system is used, the jitter will also indirectly lead to emittance growth, since the jitter disturbs the feedback BPMs and causes incorrect steering. Imperfections in the phase and gradient of the accelerating structures also need to be studied, but this has not been done in this paper.

It is of great importance to understand the effect of the different imperfections and to find efficient methods for reducing the emittance growth they cause. Efficient simulations and accurate analytic expressions facilitate the development of such methods. The simulations in this paper were all performed using the $1500 \mathrm{GeV}$ lattice described in [7]. The most important beam parameters are summarized in Table I. Throughout the paper, the term emittance refers to normalized vertical emittance. 
TABLE I. CLIC beam parameters.

\begin{tabular}{lc}
\hline \hline \multicolumn{1}{c}{ Parameter } & CLIC \\
\hline Initial energy $[\mathrm{GeV}]$ & 9 \\
Final energy [GeV] & 1500 \\
Initial vertical emittance $[\mathrm{nm}]$ & 5 \\
Particles per bunch $\left[10^{9}\right]$ & 2.56 \\
Bunch length $[\mu \mathrm{m}]$ & 30.8 \\
Pulse repetition rate $\left[\mathrm{s}^{-1}\right]$ & 150 \\
\hline \hline
\end{tabular}

In the following section, an efficient way of simulating dynamic imperfections will be described. This method will then be shown to facilitate an analytic determination of the emittance growth caused by jitter and ground motion while using a feedback system with imperfect BPMs. The analytic expressions are also confirmed by comparisons to full simulations. Finally, it will be described how the feedback system can be optimized for the optics of a certain machine and the particular ground motion spectrum of the site.

\section{EFFICIENT SIMULATIONS OF DYNAMIC IMPERFECTIONS}

In order to simulate the effect of dynamic imperfections efficiently, it is necessary to use information about the beam trajectory through the feedback BPMs and about the particle positions and angles at the end of the machine. The BPM readings will be used by the feedback system to determine the best way to steer the beam and will be represented by a column vector

$$
\mathbf{b}=\left(b_{1}, b_{2}, \ldots, b_{q}\right)^{T} .
$$

The emittance of a beam may be calculated using the beam coordinates at the end of the linac, represented by

$$
\tilde{\mathbf{y}}=\left(y_{1}, y_{2}, \ldots, y_{p}, y_{1}^{\prime}, y_{2}^{\prime}, \ldots, y_{p}^{\prime}\right)^{T},
$$

where $y_{i}$ and $y_{i}^{\prime}$ are the position and angle of macroparticle $i$. In addition to the position and angle, each macroparticle is described by its second moments and its weight $w_{i}$. During the simulations in this paper, the beam was modeled by 21 longitudinal slices each containing 7 macroparticles, i.e., in total 147 macroparticles. As a consequence, the coordinate vector $\tilde{\mathbf{y}}$ is 294-dimensional. This number of macroparticles has been seen to offer a sufficiently accurate model of the beam during various previous simulations, see for example [3].

Using PLACET [8], the response of each individual particle coordinate to different element displacements may be determined and represented by a matrix $\tilde{\mathbf{R}}$. Such a response matrix contains the coordinate changes $\Delta \tilde{\mathbf{y}}$ corresponding to each element displacement placed columnwise. Similarly, the changes of the trajectory of the beam as a whole, and consequently of the BPM readings, may be described by a response matrix. The use of matrices to represent the element displacements is validated by the fact that each coordinate of the beam is linear in terms of element displacements. Any change of the linac with this property can be represented by a response matrix. Important examples include displacements of quadrupoles and accelerating structures (generating dispersion and wakefields, consequently leading to emittance growth).

A response matrix $\tilde{\mathbf{R}}_{c s}$ was calculated to describe the coordinate (index $c$ ) change at the end of the machine caused by a unit displacement of each of the accelerating structures (index $s$ ) of the linac. A matrix $\mathbf{R}_{B s}$, containing the response of the BPM readings (index $B$ ) was also calculated. Because of the many (21914) accelerating structures of the CLIC main linac, the response matrices become rather large and computations involving them may become slow. For the simulations and calculations that follow below, all information needed is, however, contained in the 294 macroparticle coordinates (and/or in the BPM readings). The magnitude of the individual element displacements causing changes in the coordinates (and/or BPM readings) will not be used. Under the assumption that accelerating structure jitter causes displacements that are independent with the same Gaussian distributions (standard deviation $\sigma_{s}$ ), the large number of dependencies intrinsic to the $294 \times 21914$ coordinate response matrix may be removed by noting that the jitter gives rise to a coordinate change

$$
\tilde{\mathbf{y}}=\tilde{\mathbf{R}}_{c s} \mathbf{j}_{s}=\mathbf{U W} \mathbf{V}^{T} \mathbf{j}_{s},
$$

where $\tilde{\mathbf{R}}_{c s}$ has been singular value decomposed. Because of the assumptions made, the multidimensional distribution describing $\mathbf{j}_{s}$ is spherically symmetric and consequently not affected by the norm-preserving matrix $\mathbf{V}^{T}$. In addition, it should be noted that the $294 \times 21914$ matrix W contains the 294 singular values of the response matrix in the diagonal and apart from that only zeroes. The coordinates $\tilde{\mathbf{y}}$ will therefore not be affected by removing all but the first 294 columns of $\mathbf{W}$ and all but the first 294 rows of the "rotated" jitter vector $\mathbf{r}_{s}$ :

$$
\tilde{\mathbf{y}}=\mathbf{U W} \mathbf{V}^{T} \mathbf{j}_{s}=\mathbf{U W r _ { s }}=\mathbf{U} \mathbf{W}^{\prime} \mathbf{r}_{s}^{\prime}=\tilde{\mathbf{R}}_{c s}^{\prime} \mathbf{r}_{s}^{\prime} .
$$

The $294 \times 294$-matrix $\tilde{\mathbf{R}}_{c s}^{\prime}$ is definitely easier to handle than $\tilde{\mathbf{R}}_{c s}$. Notice also that the 294 components of $\mathbf{r}_{s}^{\prime}$ have the same Gaussian distribution as the initially used accelerating structure jitter.

In [3], it was described how the coordinates of a beam represented by macroparticles may be normalized with respect to their effect on emittance growth (see next section for a brief description). While coordinates and coordinate response matrices have so far been expressed in nonnormalized coordinates (indicated by a tilde), normalization offers a better understanding of how a coordinate change affects emittance. In normalized coordinates (no tilde), the emittance growth induced by a coordinate change $\mathbf{y}$ is $\Delta \epsilon_{N} \approx|\mathbf{y}|^{2}$. 


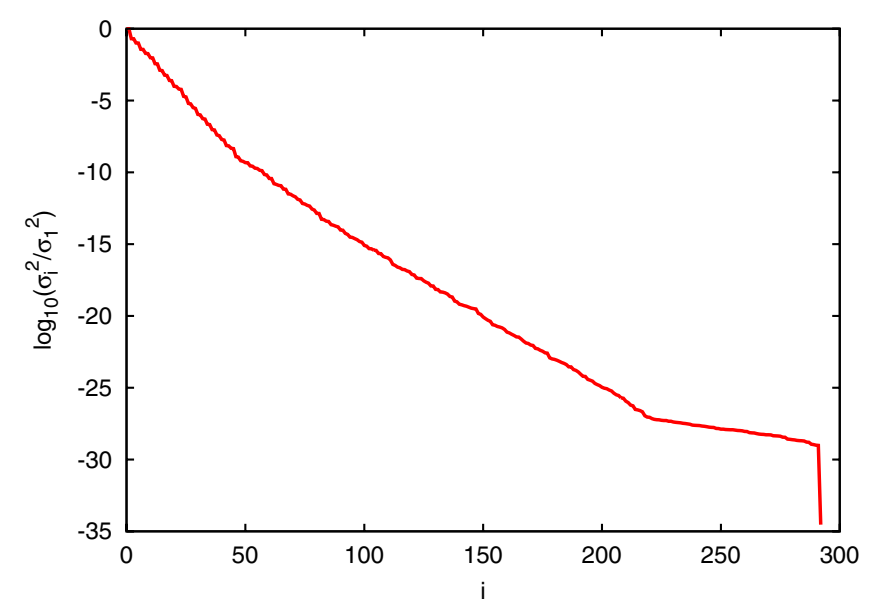

FIG. 1. (Color) Squared singular values of $\mathbf{R}_{c s}$ relative to $\sigma_{1}^{2}$. The rapid decrease indicates that only a few of the columns of $\mathbf{R}_{c s}$ will actually affect the coordinates and the emittance of the beam.

Because of the rapid decrease of the singular values $\sigma_{i}$ of the normalized response matrix $\mathbf{R}_{c s}$ (see Fig. 1), the last columns have very little effect on the normalized coordinates $\mathbf{y}$ (and consequently on the emittance). Obviously, a matrix with an even smaller number of columns may be used to accurately describe the coordinate changes caused by accelerating structure jitter. For the accelerating structure jitter, the first 15 columns should be sufficient, since these columns can be seen to represent $99.9 \%$ of the total emittance growth caused by jittering structures. In this paper, the complete $294 \times 294$-matrix was however used, since no further speed-up seemed to be necessary.

Observe that by reducing the response matrices like this, the correlation between BPM and coordinate response is lost. In some cases, e.g., ground motion (index $g$ ), this cannot be accepted, and a joint response matrix

$$
\left(\begin{array}{l}
\mathbf{R}_{c g} \\
\mathbf{R}_{B g}
\end{array}\right)
$$

has to be decomposed instead of the two matrices separately.

As a final remark regarding this way of saving computer power, it may be mentioned that the singular value decomposition of the large accelerating structure response matrix will be too memory demanding for most computers of today. In this case, the simplification of the large response matrix was carried out by decomposing a partitioned response matrix as follows:

$$
\mathbf{R}_{c s} \mathbf{j}_{s}=\left(\begin{array}{lll}
\mathbf{R}_{c s}^{1} & \cdots & \mathbf{R}_{c s}^{5}
\end{array}\right)\left(\begin{array}{c}
\mathbf{j}_{s}^{1} \\
\vdots \\
\mathbf{j}_{s}^{5}
\end{array}\right)=\left(\begin{array}{lll}
\mathbf{U}^{1} \mathbf{W}^{\prime 1} & \cdots & \mathbf{U}^{5} \mathbf{W}^{\prime 5}
\end{array}\right)\left(\begin{array}{c}
\mathbf{r}_{s}^{\prime 1} \\
\vdots \\
\mathbf{r}_{s}^{15}
\end{array}\right)=\mathbf{U W}^{\prime} \mathbf{r}^{\prime}=\mathbf{R}_{c s}^{\prime} \mathbf{r}_{s}^{\prime} .
$$

In the second step, five separate decompositions were carried out, and in the third step, one additional decomposition of a $294 \times(5 \times 294)$-matrix was performed. The resulting response matrix is consequently $294 \times 294$ as before. This procedure may obviously be generalized to response matrices with any large number of columns.

For the simulations of this paper, the following response matrices were calculated using PLACET. Even though not indicated by an apostrophe, all matrices were reduced according to the explanation above: (i) $\mathbf{R}_{c s}$-Response of beam coordinates (at the end of the machine) to structure displacements. The effect of 21914 structures on 294 coordinates is described by a $294 \times 294$-matrix. (ii) $\mathbf{R}_{B s}$-Response of BPM readings to structure displacements. This is a $1324 \times 1324$ matrix. (iii) $\mathbf{R}_{c q}-$ Response of beam coordinates to quadrupole displacements. This is a $294 \times 294$ matrix. (iv) $\mathbf{R}_{B q}$-Response of BPM readings to quadrupole displacements. This is a $1324 \times 1324$ matrix. (v) $\mathbf{R}_{c b}$-Response of beam coordinates to injected beam displacement. This is a 294component vector since it represents one single jitter source. (vi) $\mathbf{R}_{B b}$-Response of BPM readings to injected beam displacement. This is a 1324-component vector since it represents one single jitter source. (vii) $\mathbf{R}_{c g}$ - Response of beam coordinates to ground motion. Since the correlation between coordinate and BPM reading changes is important, a joint response matrix [cf. Eq. (5) and accompanying comment] was decomposed in this case. This is consequently a $294 \times 1618$-matrix. (viii) $\mathbf{R}_{B g}-$ Response of BPM readings to ground motion. By similar arguments as for $\mathbf{R}_{c g}$, this is a $1324 \times 1618$ matrix.

In the following section a more complete description of the individual matrices will be given. As will be seen, both the normalized and the non-normalized version of a response matrix is useful. By using $\mathbf{R}_{c q}$ and $\mathbf{R}_{B q}$ a response matrix describing the feedback system may also be calculated. The construction of such a response matrix will be described in more detail in Sec. III C 2.

After determination of these matrices, the effect of all the corresponding imperfections may be simulated fast and accurately using simple matrix algebra. In case even faster simulations are required, the number of columns of the matrices may be further reduced depending on the singular values. As will be shown in the following section, the 
response matrices are also easily used to make analytical predictions of the emittance growth caused by the different sources.

\section{ANALYTIC TREATMENT OF DYNAMIC IMPERFECTIONS}

\section{A. Emittance function and normalized coordinates}

As was described in detail in [3], the coordinates of the beam at the end of the linac may be normalized in terms of their effect on the emittance. In short, the emittance growth at the end of the linac may be approximated to second order by

$$
\Delta \epsilon_{N}=\epsilon_{N}-\epsilon_{N 0} \approx \frac{1}{2} \tilde{\mathbf{y}}^{T} \mathbf{H} \tilde{\mathbf{y}}
$$

where

$$
\mathbf{H}=\gamma_{r}\left(\begin{array}{cc}
\gamma \mathbf{H}^{\prime} & -\alpha \mathbf{H}^{\prime} \\
-\alpha \mathbf{H}^{\prime} & \beta \mathbf{H}^{\prime}
\end{array}\right)
$$

and

$$
\begin{aligned}
\mathbf{H}^{\prime}= & \mathbf{H}_{+}^{\prime}-\mathbf{H}_{-}^{\prime} \\
= & \left(\begin{array}{cccc}
w_{1} & 0 & \cdots & 0 \\
0 & w_{2} & \cdots & 0 \\
\vdots & \vdots & \ddots & \vdots \\
0 & 0 & \cdots & w_{n}
\end{array}\right) \\
& -\left(\begin{array}{cccc}
w_{1}^{2} & w_{1} w_{2} & \cdots & w_{1} w_{n} \\
w_{2} w_{1} & w_{2}^{2} & \cdots & w_{2} w_{n} \\
\vdots & \vdots & \ddots & \vdots \\
w_{n} w_{1} & w_{n} w_{2} & \cdots & w_{n}^{2}
\end{array}\right) .
\end{aligned}
$$

The Twiss parameters $\alpha, \beta$, and $\gamma$ are determined by the optics of the machine, $\epsilon_{N 0}$ is the emittance at the end of a perfectly aligned linac, and $\gamma_{r}$ is the Lorentz factor.

By reformulating Eq. (7) using eigenvalue decomposition, the emittance growth at the end of the linac may be approximated to second order by

$$
\Delta \epsilon_{N} \approx \tilde{\mathbf{y}}^{T} \mathbf{M}^{T} \mathbf{M} \tilde{\mathbf{y}}=\mathbf{y}^{T} \mathbf{y}
$$

and a normalization of the coordinates is obtained. The normalized coordinates are denoted $\mathbf{y}$ (as opposed to nonnormalized coordinates with a tilde). Observe that when multipulse emittance is considered in Sec. IV B, normalized coordinates cannot be used (or rather, a different normalization is necessary). This is a consequence of the fact that a uniform change of either particle positions or particle angles has no influence on single-pulse emittance, and these 2 degrees of freedom are removed through the normalization. In the multipulse case, such changes, however, contribute strongly to emittance growth and have to be taken into account.

\section{B. Jitter-induced emittance growth}

Using the notation introduced above, the emittance growth induced by different jitter sources may now be calculated. Calculations will be carried out for quadrupole, accelerating structure, and injected beam jitter, respectively. The jitter is assumed to be fast enough to appear uncorrelated on a pulse-to-pulse time scale. The trajectory feedback system will therefore be of no use for this kind of imperfection. The vibrations of any two elements are also assumed to be uncorrelated.

Element vibrations may for instance be caused by the water cooling systems, see $[9,10]$. Jitter of the beam injected in the main linac is caused by dynamic imperfections in the damping rings and the ring to main linac transfer line. A first estimate of the emittance growth caused by jitter can be obtained using response matrices and simple theoretical arguments. For simplicity it is assumed that the beam line is initially perfectly aligned. The effect of, for instance, quadrupole jitter may then be calculated using the response matrix $\mathbf{R}_{c q}$ containing the normalized coordinate response to displacements of each of the quadrupoles of the linac. It may be assumed that the matrix has been reduced in the way described in Sec. II, and the coordinate changes at the end of the linac caused by quadrupole jitter can be written

$$
\mathbf{y}=\mathbf{R}_{c q} \mathbf{r}_{q},
$$

where the components of the stochastic vector $\mathbf{r}_{q}$ have a Gaussian distribution with the same standard deviation $\sigma_{q}$ as the quadrupole jitter. According to Eq. (10), a secondorder approximation of the emittance growth caused by these coordinate changes is

$$
\Delta \epsilon_{N} \approx \mathbf{y}^{T} \mathbf{y}=\mathbf{r}_{q}^{T} \mathbf{R}_{c q}^{T} \mathbf{R}_{c q} \mathbf{r}_{q}
$$

The expectation value of the emittance growth is

$$
\left\langle\Delta \boldsymbol{\epsilon}_{N}\right\rangle \approx\left\langle\mathbf{r}_{q}^{T} \mathbf{R}_{c q}^{T} \mathbf{R}_{c q} \mathbf{r}_{q}\right\rangle=\left\langle\sum_{j k}\left[\mathbf{r}_{q}\right]_{j}\left[\mathbf{R}_{c q}^{T} \mathbf{R}_{c q}\right]_{j k}\left[\mathbf{r}_{q}\right]_{k}\right\rangle .
$$

Since the components of $\mathbf{r}_{q}$ are independent with expectation values equal to zero, terms with $j \neq k$ do not contribute, and it is found that

$$
\begin{aligned}
\left\langle\Delta \boldsymbol{\epsilon}_{N}\right\rangle & \approx \sum_{j}\left\langle\left[\mathbf{r}_{q}\right]_{j}\left[\mathbf{R}_{c q}^{T} \mathbf{R}_{c q}\right]_{j j}\left[\mathbf{r}_{q}\right]_{j}\right\rangle=\sigma_{q}^{2} \operatorname{Tr}\left(\mathbf{R}_{c q}^{T} \mathbf{R}_{c q}\right) \\
& =\sigma_{q}^{2}\left\|\mathbf{R}_{c q}\right\|_{F}^{2} .
\end{aligned}
$$

In the second step, the variance $\sigma_{q}^{2}$ of the stochastic vector components was used. Observe that the trace of the symmetric product $\mathbf{R}_{c q}^{T} \mathbf{R}_{c q}$ is most efficiently calculated as the squared Frobenius norm $\left(\left\|\mathbf{R}_{c q}\right\|_{F}^{2}\right)$ [11] of $\mathbf{R}_{c q}$, i.e., as the sum of the squares of each matrix component.

An alternative way of calculating the expectation value is to use the eigenvalue decomposition of $\mathbf{R}_{c q}^{T} \mathbf{R}_{c q}$ : 


$$
\begin{aligned}
\left\langle\Delta \epsilon_{N}\right\rangle & \approx\left\langle\mathbf{r}_{q}^{T} \mathbf{R}_{c q}^{T} \mathbf{R}_{c q} \mathbf{r}_{q}\right\rangle=\left\langle\mathbf{r}_{q}^{T} \mathbf{Q D Q} \mathbf{Q}^{T} \mathbf{r}_{q}\right\rangle \\
& =\left\langle\left(\mathbf{r}_{q}^{\prime}\right)^{T} \mathbf{D} \mathbf{r}_{q}^{\prime}\right\rangle=\left\langle\sum_{j} d_{j}\left[\mathbf{r}_{q}^{\prime}\right]_{j}^{2}\right\rangle=\sigma_{q}^{2} \sum_{j} d_{j} .
\end{aligned}
$$

Since the sum of the eigenvalues of a matrix equals its trace, this is the same result as in Eq. (13).

Equations (13) and (14) clearly show that the emittance growth caused by quadrupole jitter scales quadratically with the standard deviation $\sigma_{q}$ of the Gaussian jitter distribution. For the case of the CLIC main linac the squared Frobenius norm of the response matrix leads to a scaling factor $e_{q} \approx 8.69 \times 10^{4} \mathrm{~nm} / \mu \mathrm{m}^{2}$ :

$$
\left\langle\Delta \epsilon_{N}\right\rangle \approx \sigma_{q}^{2}\left\|\mathbf{R}_{c q}\right\|_{F}^{2}=\sigma_{q}^{2} e_{q} .
$$

Similarly the expression

$$
\left\langle\Delta \epsilon_{N}\right\rangle \approx \sigma_{s}^{2}\left\|\mathbf{R}_{c s}\right\|_{F}^{2}=\sigma_{s}^{2} e_{s}
$$

describes the emittance growth caused by accelerating structure jitter with a response matrix $\mathbf{R}_{c s}$. The scaling factor in this case is $e_{s} \approx 0.224 \mathrm{~nm} / \mu \mathrm{m}^{2}$. Obviously the emittance in the linac is much more sensitive to quadrupole jitter.

Jitter in the vertical position of the injected beam may be treated in the same way, and since there is only one jitter source in this case, the determination of the scaling factor is particularly straightforward:

$$
\left\langle\Delta \epsilon_{N}\right\rangle \approx \sigma_{b}^{2}\left\|\mathbf{R}_{c b}\right\|_{F}^{2}=\sigma_{b}^{2}\left\|\mathbf{R}_{c b}\right\|^{2}=\sigma_{b}^{2} e_{b} .
$$

As mentioned in Sec. II, the injected beam response matrix has one single column. The scaling factor is obviously equal to the emittance growth caused by a unit displacement of the injected beam, i.e. $e_{b} \approx 5.13 \mathrm{~nm} / \mu \mathrm{m}^{2}$.

In order to confirm the analytical results, each jitter source was studied by tracking 9000 pulses (corresponding to 1 min of operation) through the CLIC main linac using PLACET. The jitter was simulated by randomly modifying the injected beam position, each quadrupole position, or each accelerating structure position between pulses. Figure 2 shows the excellent agreement of the predictions and the simulations. In particular for jitter leading to low emittance growth, the agreement is nearly exact. For stronger jitter, and consequently more emittance growth, the second-order emittance approximation tends to yield an overestimation of the emittance growth.

Observe that the emittance growth caused by several of the sources simultaneously is obtained by simply summing the different contributions. This is easily realized by noting that

$$
\begin{aligned}
\left\langle\Delta \epsilon_{N}\right\rangle & \approx\left\langle\left(\mathbf{y}_{1}+\mathbf{y}_{2}\right)^{T}\left(\mathbf{y}_{1}+\mathbf{y}_{2}\right)\right\rangle \\
& =\left\langle\mathbf{y}_{1}^{T} \mathbf{y}_{1}\right\rangle+\left\langle\mathbf{y}_{2}^{T} \mathbf{y}_{2}\right\rangle+2\left\langle\mathbf{y}_{1}^{T} \mathbf{y}_{2}\right\rangle,
\end{aligned}
$$

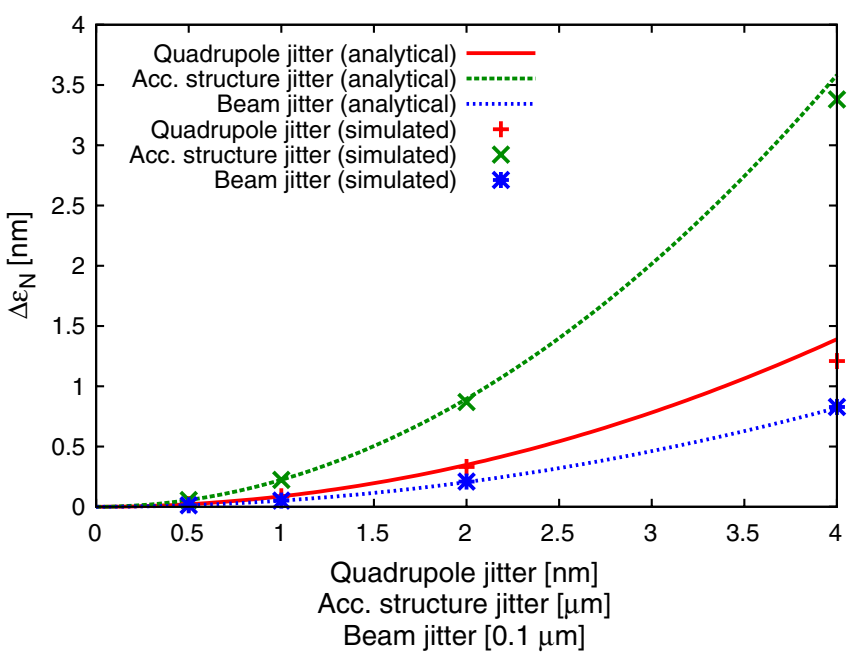

FIG. 2. (Color) Jitter-induced emittance growth according to analytic estimates and simulations, respectively.

where the first two terms are the individual contributions calculated as above. The third term is a mixed term and is zero since

$$
\left\langle\mathbf{y}_{1}^{T} \mathbf{y}_{2}\right\rangle=\left\langle\mathbf{r}_{1}^{T} \mathbf{R}_{1}^{T} \mathbf{R}_{2} \mathbf{r}_{2}\right\rangle=\left\langle\sum_{j k}\left[\mathbf{r}_{1}\right]_{j}\left[\mathbf{R}_{1}^{T} \mathbf{R}_{2}\right]_{j k}\left[\mathbf{r}_{2}\right]_{k}\right\rangle=0,
$$

where the fact that the stochastic vectors $\mathbf{r}_{1}$ and $\mathbf{r}_{2}$ are independent with zero expectation value was used in the last step. The total emittance growth caused by the jitter effects described above is therefore

$$
\left\langle\Delta \epsilon_{N}\right\rangle \approx \sigma_{q}^{2} e_{q}+\sigma_{s}^{2} e_{s}+\sigma_{b}^{2} e_{b} .
$$

Note that, if $\mathbf{y}_{1}$ in Eq. (18) is caused by an initial (static) misalignment of the machine instead of by a jitter source, the total expected emittance growth will be the sum of the initial emittance growth and the same jitter contribution as expected for an aligned machine.

Since jitter makes the beam position (and angle) vary rapidly from one pulse to another, without the possibility for a feedback system to correct it, single-pulse emittance is not the most relevant parameter for maximization of luminosity. As will be described in detail in Sec. IV B, a better measure may instead be the so-called multipulse emittance, i.e., the emittance of an ensemble of consecutive pulses.

\section{Ground motion and trajectory feedback}

One very severe dynamic imperfection is caused by ground motion. If ground motion is not corrected, the emittance will keep growing and quickly become unacceptably large. Several previous CLIC studies have shown that a trajectory feedback system can be used to prevent the emittance growth caused by ground motion $[4,5]$. 
Similarly, trajectory feedback systems have been investigated in the context of SLC and NLC $[12,13]$.

\section{Ground motion}

Ground motion can be modeled in several ways. The simplest model may be the so-called ATL model $[6,14]$. According to this model the variance of the relative (vertical) displacement of two elements equals

$$
\left(\sigma_{\Delta y}\right)^{2}=A \times T \times L,
$$

where $A$ is a site-specific parameter with units $\mu \mathrm{m}^{2} / \mathrm{s} / \mathrm{m}$, the (longitudinal) distance between the elements is denoted $L$, and the time since the elements were aligned is $T$. The $A T L$ model parameter was in this paper chosen, like in [4], to be $A=0.5 \times 10^{-6} \mu \mathrm{m}^{2} / \mathrm{s} / \mathrm{m}$. This value was obtained from stability measurements in the SLAC tunnel [15].

To determine the absolute girder displacements caused by ground motion, according to the ATL model, the following simple matrix operation may be carried out:

$$
\mathbf{j}=\left(\begin{array}{cccc}
\sigma_{1} & 0 & 0 & \cdots \\
\sigma_{1} & \sigma_{2} & 0 & \cdots \\
\sigma_{1} & \sigma_{2} & \sigma_{3} & \cdots \\
\vdots & \vdots & \vdots & \ddots
\end{array}\right) \mathbf{r}^{\prime}=\mathbf{C}^{T} \mathbf{r}^{\prime}
$$

Here, $\mathbf{r}^{\prime}$ is a vector of random components with Gaussian distribution and standard deviation 1 . The sigmas are determined by $\sigma_{i}=\sqrt{A \times T \times \Delta L_{i, i-1}}$, where the distance $\Delta L_{i, i-1}$ between two consecutive girders has been used. Consequently, $r_{i}=\sigma_{i} r_{i}^{\prime}$ represents the random relative displacement of the two girders at a time $T$. In the CLIC main linac, the girders are equally spaced with a distance of $2.23 \mathrm{~m}$. As seen in Table I, the pulse repetition rate is $150 \mathrm{~s}^{-1}$ and, as a consequence, the time $T$ between two pulses is $1 / 150 \mathrm{~s}$. Apparently, all $r_{i}$ have the same Gaussian distribution with

$$
\begin{aligned}
\sigma_{\mathrm{gm}} & =\sqrt{A \times T \times \bar{X}}=\sqrt{0.5 \times 10^{-6} \times \frac{1}{150} \times 2.23} \mu \mathrm{m} \\
& \approx 8.622 \times 10^{-5} \mu \mathrm{m} .
\end{aligned}
$$

Between two pulses, ground motion causes a coordinate change

$$
\mathbf{y}=\mathbf{R}_{c g, \mathrm{abs}} \mathbf{C}^{T} \mathbf{r}^{\prime}=\mathbf{R}_{c g} \mathbf{r},
$$

where a response matrix $\mathbf{R}_{c g \text {,abs }}$, representing the response of absolute girder displacements, has been used. Similarly,

$$
\mathbf{b}=\mathbf{R}_{B g, \mathrm{abs}} \mathbf{C}^{T} \mathbf{r}^{\prime}=\mathbf{R}_{B g} \mathbf{r} .
$$

After $P$ pulses, the total coordinate change caused by ground motion is

$$
\mathbf{y}_{P}=\sum_{j=0}^{P-1} \mathbf{R}_{c g} \mathbf{r}_{j},
$$

where $\mathbf{r}_{j}$ is a random vector representing the change in relative girder displacements between pulse $j$ and pulse $j+1$. The $\mathbf{r}_{j}$ are all independent, and by using similar arguments as before [cf. Eqs. (12), (13), (18), and (19)], the expected emittance growth after $P$ pulses can be shown to be

$$
\begin{aligned}
\left\langle\Delta \epsilon_{N}\right\rangle & \approx\left\langle\mathbf{y}_{P}^{T} \mathbf{y}_{P}\right\rangle=\left\langle\left(\sum_{j=0}^{P-1} \mathbf{R}_{c g} \mathbf{r}_{j}\right)^{T}\left(\sum_{j=0}^{P-1} \mathbf{R}_{c g} \mathbf{r}_{j}\right)\right\rangle \\
& =\sum_{j=0}^{P-1}\left\langle\mathbf{r}_{j}^{T} \mathbf{R}_{c g}^{T} \mathbf{R}_{c g} \mathbf{r}_{j}\right\rangle=P \sigma_{\mathrm{gm}}^{2} e_{\mathrm{gm}},
\end{aligned}
$$

where

$$
e_{\mathrm{gm}}=\left\|\mathbf{R}_{c g}\right\|_{F}^{2} .
$$

For the CLIC main linac lattice used here, the scaling factor is $e_{\mathrm{gm}} \approx 2.37 \times 10^{5} \mathrm{~nm} / \mu \mathrm{m}^{2}$. By inserting this value together with Eq. (23) into Eq. (27), the emittance growth rate is seen to be approximately $1.76 \times$ $10^{-3} \mathrm{~nm} /$ pulse $(0.264 \mathrm{~nm} / \mathrm{s})$ if the ground motion effects are not counteracted. This rapid increase means that the second-order emittance growth approximation quickly becomes inaccurate if no feedback is used.

\section{Trajectory feedback}

The trajectory feedback system used to control ground motion effects was first chosen according to [4,5]. In total 40 feedbacks were used to locally resteer the beam to the reference trajectory, which is defined to be the trajectory of the beam after the initial beam-based alignment. Each feedback consisted of two dipole correctors and three BPMs. The dipoles were located in two consecutive vertically focusing quadrupoles (and were simulated as displacements of the quadrupoles). The first of the BPMs was located in front of the second quadrupole and the other two in front of the two following focusing quadrupoles. The feedbacks were assumed to be dependent, i.e., they could exchange information.

The effect of applying the feedbacks can be determined by first calculating a BPM response matrix $\mathbf{R}_{B d}$ where a column contains the change in BPM readings (beam position) for a unit change of a dipole corrector (index $d$ ). The response matrix for the feedback system described above is consequently a $120 \times 80$ matrix, and by using this matrix the beam position change for a corrector change $\Delta \mathbf{d}$ can be written $\mathbf{R}_{B d} \Delta \mathbf{d}$. If the beam position in each BPM is 
denoted by the vector $\mathbf{b}$, the optimal corrector changes can be found by solving

$$
\mathbf{b}+\mathbf{R}_{B d} \Delta \mathbf{d}=0 .
$$

Since this equation system is overdetermined, in general no exact solution exists and a least-square solution is instead obtained through

$$
\Delta \mathbf{d}=-\left[\mathbf{R}_{B d}^{T} \mathbf{R}_{B d}\right]^{-1} \mathbf{R}_{B d}^{T} \mathbf{b}=\mathbf{R}_{d B} \mathbf{b},
$$

where a new response matrix $\mathbf{R}_{d B}$ has been introduced to describe how the dipole correctors should ideally respond to a certain BPM reading $\mathbf{b}$. Because of various imperfections, e.g. finite BPM resolution, the feedbacks may perform better if only a fraction (gain) $g$ of the calculated change $\Delta \mathbf{d}$ is applied before the next pulse. By using the coordinate response matrix $\mathbf{R}_{c d}$ for the effect of a dipole corrector change, it is possible to express the effect that a certain nonzero BPM reading has as

$$
\Delta \mathbf{y}=g \mathbf{R}_{c d} \mathbf{R}_{d B} \mathbf{b}=g \mathbf{R}_{c B} \mathbf{b},
$$

for a feedback system with gain $g$. Similarly the BPM response matrix $\mathbf{R}_{B d}$ may be used to obtain

$$
\Delta \mathbf{b}=g \mathbf{R}_{B d} \mathbf{R}_{d B} \mathbf{b}=g \mathbf{R}_{B B} \mathbf{b},
$$

describing how the BPM readings will change as a consequence of the feedback steering induced by a nonzero BPM reading $\mathbf{b}$. This formulation of the feedback system facilitates the study of emittance growth caused by ground motion and by the feedback system itself.

\section{Emittance growth caused by ground motion and trajectory feedback}

As can be seen in Fig. 3, ground motion effects rapidly lead to unacceptable emittance growth. The emittance growth is significantly reduced by the basic trajectory feedback system described above. However, steering the beam close to the centers of the feedback BPMs does not necessarily minimize emittance growth, and some emittance growth is inevitable. By using high gain, the inevitable emittance growth may be kept at a minimum. For $g=1$, only the effect of ground motion between the two last pulses will be left uncorrected. For a lower gain on the other hand, earlier ground motion effects will also be partly uncorrected and contribute to emittance growth. However, as can be seen in Fig. 3, a gain of as little as $g=0.01$ may be sufficient to achieve acceptable emittance preservation. Observe that the rate with which emittance growth increases with time (the slope in Fig. 3) does not depend on the gain. Instead, reduced gain leads to a constant (timeindependent) additional emittance growth.

If the resolution of the BPMs used for feedback is also taken into account, the results are different. If the gain is too high, the errors in the BPM readings cause incorrect steering of a magnitude such that the performance of the feedback system is deteriorated and a lower gain may be

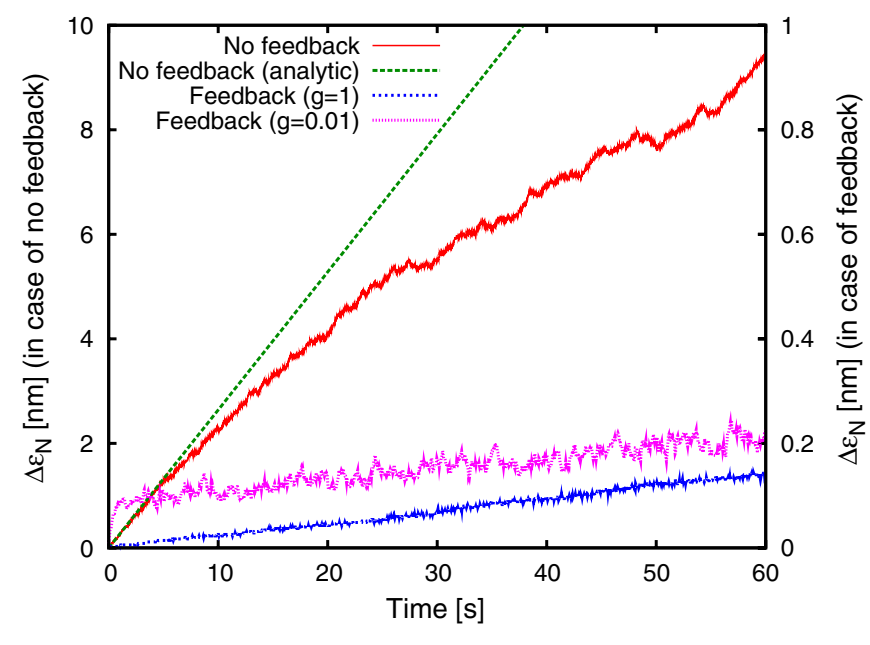

FIG. 3. (Color) Emittance growth caused by ground motion averaged over 100 machines. Observe that the left emittance scale applies when feedback is not used, and the right scale applies to the results obtained using feedback. Without feedback, the emittance quickly gets unacceptably large. During the first seconds, the emittance growth rate is in excellent agreement with the value $(0.264 \mathrm{~nm} / \mathrm{s})$ derived in Sec. III C 1 . Since the emittance growth quickly becomes large, the second-order emittance approximation is however only accurate for a few seconds. By using feedback with gain $g=1$, or even as low as $g=0.01$, the emittance may be reasonably well preserved.

more appropriate. According to [4], the optimal gain for this kind of feedback system is 0.02 if a BPM resolution of $0.1 \mu \mathrm{m}$ is assumed. Because of a few differences between the machine parameters, the optimization of the gain was, however, performed again. As will be shown below, the optimal gain is higher in this case, even though not only BPM resolution but also a few additional imperfections were taken into account.

The emittance growth caused by a BPM resolution $\sigma_{\mathrm{BPM}}$ for a feedback with gain $g$ may be estimated theoretically by studying the feedback response matrix $\mathbf{R}_{c B}$ in a similar way as for the treatment of jitter effects in Sec. III B. Once again, it is assumed that the machine is initially aligned. The emittance growth is in this case caused by the finite BPM resolution that makes the feedback steer the beam incorrectly. By denoting the incorrect part of the BPM readings at pulse $i(i=0$ corresponding to the most recent) by $\mathbf{b}_{i}$, Eqs. (31) and (32) may be used to determine the effect of the finite BPM resolution. The feedback itself will correct the undesired steering during the following pulses, but will only remove a fraction $g$ per pulse. After $i$ pulses, a coordinate change $(1-g)^{i} \Delta \mathbf{y}$ still remains as a consequence of the incorrect BPM reading. By summing contributions from all incorrect BPM readings during the last $P$ pulses, a total coordinate error

$$
\mathbf{y}=\sum_{i=0}^{P-1}(1-g)^{i} g \mathbf{R}_{c B} \mathbf{b}_{i}=g \mathbf{R}_{c B} \sum_{i=0}^{P-1}(1-g)^{i} \mathbf{b}_{i}
$$


is obtained. The emittance growth caused by this error is

$$
\begin{aligned}
\left\langle\Delta \epsilon_{N}\right\rangle & \approx\left\langle\mathbf{y}^{T} \mathbf{y}\right\rangle \\
& =g^{2}\left\langle\left[\sum_{i=0}^{P-1}(1-g)^{i} \mathbf{b}_{i}\right]^{T} \mathbf{R}_{c B}^{T} \mathbf{R}_{c B} \sum_{i=0}^{P-1}(1-g)^{i} \mathbf{b}_{i}\right\rangle \\
& =g^{2} \sum_{i=0}^{P-1}(1-g)^{2 i}\left\langle\mathbf{b}_{i}^{T} \mathbf{R}_{c B}^{T} \mathbf{R}_{c B} \mathbf{b}_{i}\right\rangle \\
& =g^{2} \sigma_{\mathrm{BPM}}^{2}\left\|\mathbf{R}_{c B}\right\|_{F}^{2} \sum_{i=0}^{P-1}(1-g)^{2 i} \\
& =g^{2} \sigma_{\mathrm{BPM}}^{2}\left\|\mathbf{R}_{c B}\right\|_{F}^{2} \frac{\left[1-(1-g)^{2 P}\right]}{g(2-g)} \\
& \approx \sigma_{\mathrm{BPM}}^{2} \frac{g}{2-g} e_{f b}
\end{aligned}
$$

for large $P$. Note that in the third step, all terms containing $\mathbf{b}_{i}^{T}$ and $\mathbf{b}_{j}$ with $i \neq j$ were removed. Such terms do not contribute to the expectation value of the emittance growth since the BPM errors are independent from one pulse to the other [cf. Eq. (19)]. Apart from the drift of the BPMs caused by ground motion, BPM errors that are correlated from pulse to pulse, e.g. systematic errors and drifting BPM readings (due to, e.g., electronics), were not considered in this paper.

The scaling factor

$$
e_{f b}=\left\|\mathbf{R}_{c B}\right\|_{F}^{2}
$$

was for this particular feedback design calculated to be $e_{f b} \approx 14.9 \mathrm{~nm} / \mu \mathrm{m}^{2}$. Observe that this source of emittance growth cannot be added to the jitter contributions in the same way as in Eq. (20). The reason is that the jitter causes the feedback system to steer incorrectly which leads to an additional indirect source of emittance growth. In order to determine the effect of, e.g., quadrupole jitter and a feedback system with gain $g$, the following expression has to be used:

$$
\mathbf{y}=\mathbf{R}_{c q} \mathbf{j}_{q}+\sum_{i=0}^{N-1}(1-g)^{i} g \mathbf{R}_{c B} \mathbf{R}_{B q} \mathbf{j}_{q, i}
$$

Here, the feedback BPMs are assumed to be perfect $\left(\sigma_{\mathrm{BPM}}=0\right)$. With a finite BPM resolution, the only modification would be the addition of the term in Eq. (33). The first term of Eq. (36) is the direct coordinate change caused by the most recent quadrupole jitter $\mathbf{j}_{q}$, while the second term is an indirect effect due to quadrupole jitter $\mathbf{j}_{q, i}$ at previous pulses leading to fluctuations in the BPM readings and consequently to incorrect steering. The effect of quadrupole jitter on beam position in the feedback BPMs is calculated using the response matrix $\mathbf{R}_{B q}$. In analogy with previous calculations of expected emittance growth, Eq. (36) leads to

$$
\begin{aligned}
\left\langle\Delta \epsilon_{N}\right\rangle & \approx \sigma_{q}^{2} e_{q}+\sigma_{q}^{2} \frac{g}{2-g}\left\|\mathbf{R}_{c B} \mathbf{R}_{B q}\right\|_{F}^{2} \\
& =\sigma_{q}^{2} e_{q}+\sigma_{q}^{2} \frac{g}{2-g} e_{f b, q}
\end{aligned}
$$

where $e_{f b, q} \approx 8.92 \times 10^{4} \mathrm{~nm} / \mu \mathrm{m}^{2}$. Observe that since the two terms in Eq. (36) contain independent stochastic variables, a third mixed term is not necessary in Eq. (37), cf. Eq. (19). Similar calculations lead to $e_{f b, s} \approx$ $0.460 \mathrm{~nm} / \mu \mathrm{m}^{2}$ for the combined effect of feedback and accelerating structure jitter and $e_{f b, b} \approx 4.97 \mathrm{~nm} / \mu \mathrm{m}^{2}$ for the combined effect of feedback and injected beam jitter.

In Sec. III C 1, the emittance growth caused by uncontrolled ground motion was calculated. In case a feedback system is used to counteract this emittance growth, the derivations have to be modified. By using Eq. (25) and the feedback response matrix $\mathbf{R}_{c B}$, it is easily seen that the feedback response to a relative girder displacement $\mathbf{r}$ is

$$
\mathbf{y}=\mathbf{R}_{c B} \mathbf{R}_{B g} \mathbf{r}
$$

for gain $g=1$. The feedback system performs this correction immediately after the pulse during which the BPMs were read out. If the gain is less than one, a smoother correction (during a large number of pulses) will instead be carried out. After $i$ pulses, the total coordinate change caused by the feedback system will then be

$$
\mathbf{y}_{f b}=\left[1-(1-g)^{i}\right] \mathbf{R}_{c B} \mathbf{R}_{B g} \mathbf{r} .
$$

As long as $g \neq 0$, this expression is seen to approach Eq. (38) as $i \rightarrow \infty$.

By using Eqs. (24) and (39), the total coordinate change caused by the relative girder displacement $\mathbf{r}$, counteracted by a feedback system (gain $g$ ), can be shown to be

$$
\mathbf{y}_{i}=\left[\mathbf{R}_{c g}+\mathbf{R}_{c B} \mathbf{R}_{B g}-(1-g)^{i} \mathbf{R}_{c B} \mathbf{R}_{B g}\right] \mathbf{r},
$$

after $i$ pulses. By summing up the contributions from all relative girder displacements $\mathbf{r}_{i}$ during the last $P$ pulses, the following expression is obtained:

$$
\begin{aligned}
\mathbf{y} & =\sum_{i=0}^{P-1}\left[\mathbf{R}_{c g}+\mathbf{R}_{c B} \mathbf{R}_{B g}-(1-g)^{i} \mathbf{R}_{c B} \mathbf{R}_{B g}\right] \mathbf{r}_{i} \\
& =\sum_{i=0}^{P-1}\left[\mathbf{R}_{S}-(1-g)^{i} \mathbf{R}_{D}\right] \mathbf{r}_{i}
\end{aligned}
$$

where two auxiliary matrices

$$
\begin{gathered}
\mathbf{R}_{D}=\mathbf{R}_{c B} \mathbf{R}_{B g}, \\
\mathbf{R}_{S}=\mathbf{R}_{c g}+\mathbf{R}_{c B} \mathbf{R}_{B g}
\end{gathered}
$$

have been introduced. The expected emittance growth may now be calculated as before. Observe that all $\mathbf{r}_{i}$ are independent stochastic variables, and no "mixed terms" contribute to the expectation value: 


$$
\begin{aligned}
\left\langle\Delta \epsilon_{N}\right\rangle \approx & \left\langle\mathbf{y}^{T} \mathbf{y}\right\rangle=\sum_{i=0}^{P-1}\left[\left\langle\mathbf{r}_{i}^{T} \mathbf{R}_{S}^{T} \mathbf{R}_{S} \mathbf{r}_{i}\right\rangle-(1-g)^{i}\left\langle\mathbf{r}_{i}^{T} \mathbf{R}_{S}^{T} \mathbf{R}_{D} \mathbf{r}_{i}\right\rangle\right. \\
& \left.-(1-g)^{i}\left\langle\mathbf{r}_{i}^{T} \mathbf{R}_{D}^{T} \mathbf{R}_{S} \mathbf{r}_{i}\right\rangle+(1-g)^{2 i}\left\langle\mathbf{r}_{i}^{T} \mathbf{R}_{D}^{T} \mathbf{R}_{D} \mathbf{r}_{i}\right\rangle\right] .
\end{aligned}
$$

This expression is easily simplified by using Frobenius norms and in case of the second and third terms, which contain nonsymmetric products, by using matrix trace. Note that since $\operatorname{Tr}(\mathbf{A})=\operatorname{Tr}\left(\mathbf{A}^{T}\right)$, the second and third terms contribute equally. Evaluation of the geometric sums then gives

$$
\begin{aligned}
\left\langle\Delta \epsilon_{N}\right\rangle \approx & \sigma_{\mathrm{gm}}^{2}\left[P e_{\mathrm{gm}, 0}-2 \frac{1-(1-g)^{P}}{g} e_{\mathrm{gm}, 1}\right. \\
& \left.+\frac{1-(1-g)^{2 P}}{g(2-g)} e_{\mathrm{gm}, 2}\right] \\
\approx & \sigma_{\mathrm{gm}}^{2}\left[P e_{\mathrm{gm}, 0}-\frac{2}{g} e_{\mathrm{gm}, 1}+\frac{1}{g(2-g)} e_{\mathrm{gm}, 2}\right]
\end{aligned}
$$

where transient terms have been removed in the last step under the assumption that $P$ is large. The new scaling factors are

$$
\begin{array}{r}
e_{\mathrm{gm}, 0}=\left\|\mathbf{R}_{S}\right\|_{F}^{2} \approx 2.29 \times 10^{3} \mathrm{~nm} / \mu \mathrm{m}^{2}, \\
e_{\mathrm{gm}, 1}=\operatorname{Tr}\left(\mathbf{R}_{S}^{T} \mathbf{R}_{D}\right) \approx 2.43 \times 10^{3} \mathrm{~nm} / \mu \mathrm{m}^{2}, \\
e_{\mathrm{gm}, 2}=\left\|\mathbf{R}_{D}\right\|_{F}^{2} \approx 2.39 \times 10^{5} \mathrm{~nm} / \mu \mathrm{m}^{2} .
\end{array}
$$

Observe that according to Eq. (45), the emittance growth per pulse equals $\sigma_{\mathrm{gm}}^{2} e_{\mathrm{gm}, 0}$, and for best possible feedback performance, the squared Frobenius norm of $\mathbf{R}_{S}$ [see Eq. (43)] should be as small as possible. By using these expressions, the emittance growth per pulse was calculated to be

$$
\frac{d\left\langle\Delta \epsilon_{N}\right\rangle}{d P} \approx 1.70 \times 10^{-5} \mathrm{~nm}
$$

for the feedback system described above. In more convenient units the emittance growth rate is, consequently, $0.153 \mathrm{~nm} /$ minute.

If $\mathbf{R}_{S} \approx 0$, then $\mathbf{R}_{D} \approx-\mathbf{R}_{c g}$ [see Eqs. (42) and (43)], and the expected emittance growth can be approximated by the third term of Eq. (45) [with $e_{\mathrm{gm}, 2} \approx e_{\mathrm{gm}}$ cf. Eq. (28)]. This term grows rapidly as $g$ is decreased and, consequently, sets a lower limit for the gain.

By summing the emittance growth caused by ground motion, feedback (gain $g$ ) with imperfect BPMs, and jitter (direct and indirect effects), a complete emittance growth expression is obtained:
TABLE II. Magnitudes assumed for the dynamic imperfections. The ground motion parameter was, as described in Sec. III C, obtained under the assumption that the ATL model parameter $A=0.5 \times 10^{-6} \mu \mathrm{m}^{2} / \mathrm{s} / \mathrm{m}$.

\begin{tabular}{lc}
\hline \hline \multicolumn{1}{c}{ Imperfection } & Magnitude \\
\hline Quadrupole jitter $\left(\sigma_{q}\right)$ & $1 \mathrm{~nm}$ \\
Accelerating structure jitter $\left(\sigma_{s}\right)$ & $1 \mathrm{~nm}$ \\
Injected beam jitter $\left(\sigma_{b}\right)$ & $0.1 \mu \mathrm{m}$ \\
BPM resolution $\left(\sigma_{\mathrm{BPM}}\right)$ & $0.1 \mu \mathrm{m}$ \\
Ground motion $\left(\sigma_{\mathrm{gm}}\right)$ & $8.622 \times 10^{-5} \mu \mathrm{m}$ \\
\hline \hline
\end{tabular}

$$
\begin{aligned}
\left\langle\Delta \epsilon_{N}\right\rangle \approx & \sigma_{q}^{2} e_{q}+\sigma_{s}^{2} e_{s}+\sigma_{b}^{2} e_{b}+\frac{g}{2-g}\left(\sigma_{\mathrm{BPM}}^{2} e_{f b}+\sigma_{q}^{2} e_{f b, q}\right. \\
& \left.+\sigma_{s}^{2} e_{f b, s}+\sigma_{b}^{2} e_{f b, b}\right)+\sigma_{\mathrm{gm}}^{2}\left(P e_{\mathrm{gm}, 0}-\frac{2}{g} e_{\mathrm{gm}, 1}\right. \\
& \left.+\frac{1}{g(2-g)} e_{\mathrm{gm}, 2}\right)
\end{aligned}
$$

This expression is accurate when the emittance growth is small enough for the second-order emittance approximation to be valid, and as long as $P$ is not small enough for the transient terms to be non-negligible. The emittance growth can be divided into three parts: a constant contribution, a constant gain-dependent contribution, and a part increasing with time. Clearly, the emittance growth may be kept at a minimum by choosing the gain $g$ wisely. The gain was optimized for imperfections according to Table II. The sum of the two constant contributions to emittance growth was calculated for different gains, see Fig. 4. The optimal gain is seen to be approximately 0.07 . The theoretical optimum was determined to be $g=0.074$, but any gain between 0.05 and 0.1 seems to lead to similar performance.

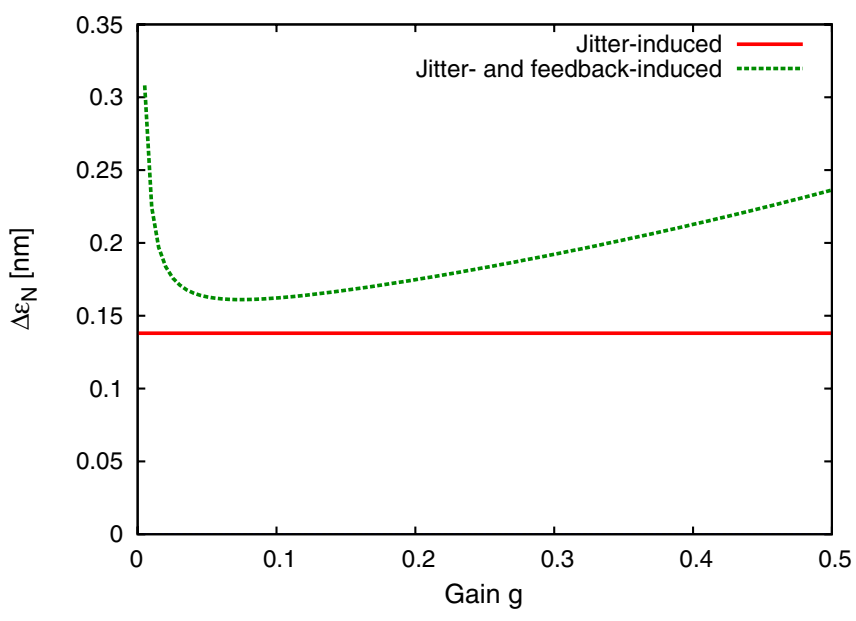

FIG. 4. (Color) Determination of the optimal feedback gain when all dynamic imperfections are considered. The optimum gain is 0.074 leading to a constant emittance growth contribution of $0.161 \mathrm{~nm}$. The jitter effect alone causes an emittance growth of $0.138 \mathrm{~nm}$. 


\section{IMPROVED FEEDBACK DESIGN}

As explained in the previous section, the feedback system is optimal if $e_{\mathrm{gm}, 0}$, and consequently $\mathbf{R}_{S}$, equals zero. This would mean that any coordinate change caused by ground motion could be perfectly corrected using only information from the feedback BPMs. For this to be possible, the response of coordinate and BPM readings to ground motion must be strongly correlated. The fact that the basic feedback design works as well as it does, clearly shows that this is indeed the case. In order to design an improved feedback system, the feedback response matrix $\mathbf{R}_{c B}$ will be optimized to minimize $e_{\mathrm{gm}, 0}$. Each column of $\mathbf{R}_{C B}$ describes how the feedback will change the beam coordinates at the end of the machine for a unit reading in the corresponding BPM. Consequently, an optimized response matrix describes the ideal coordinate response of any feedback system, without any details about how to construct such feedbacks.

The optimal $\mathbf{R}_{c B}$ is obtained by minimizing

$$
\epsilon_{g}=\frac{d\left\langle\Delta \epsilon_{N}\right\rangle}{d P} \approx \sigma_{\mathrm{gm}}^{2}\left\|\mathbf{R}_{S}\right\|_{F}^{2}=\sigma_{\mathrm{gm}}^{2}\left\|\mathbf{R}_{c g}+\mathbf{R}_{c B} \mathbf{R}_{B g}\right\|_{F}^{2},
$$

where the definition of $\mathbf{R}_{S}$ [Eq. (43)] was used. Minimization of this expression is easily performed by first noting that the squared Frobenius norm of a matrix is the sum of the squares of each matrix element. The sum for each matrix row may be minimized by solving a leastsquare problem with the unknown being the row elements of $\mathbf{R}_{c B}$. The solution for each row $i$ is

$$
\left[\mathbf{R}_{c B}\right]_{i}=-\left[\mathbf{R}_{c g}\right]_{i} \mathbf{R}_{B g}^{-1},
$$

where $\mathbf{R}_{B g}^{-1}$ is the pseudoinverse of $\mathbf{R}_{B g}$ :

$$
\mathbf{R}_{B g}^{-1}=\mathbf{V} \mathbf{W}^{-1} \mathbf{U}^{T} .
$$

The matrices $\mathbf{U}, \mathbf{W}$, and $\mathbf{V}$ are obtained by singular value decomposition of $\mathbf{R}_{B g}$ :

$$
\mathbf{R}_{B g}=\mathbf{U W V}^{T} \text {. }
$$

By merging the rows of Eq. (52), the optimal $\mathbf{R}_{c B}$ becomes

$$
\mathbf{R}_{c B}=-\mathbf{R}_{c g} \mathbf{R}_{B g}^{-1},
$$

which, upon insertion into Eq. (51), shows symbolically that the growth rate is zero. Since $\mathbf{R}_{B g}$ does not have a proper inverse, the rate will however not be exactly zero. By determining the optimal $\mathbf{R}_{c B}$ according to Eq. (55), it is seen that the growth rate may be reduced to $\epsilon_{g} \approx$ $0.037 \mathrm{~nm} /$ minute if the same 120 BPMs as in Sec. IIIC 2 are used. Observe that this is what can be achieved in theory, the construction of a feedback system with optimal response will be described in the next section.

By using all 1324 BPMs of the CLIC main linac, the performance can be significantly improved. In case of a basic trajectory feedback design as described in Sec. III C 2, with the same 80 dipole correctors, the emittance growth rate was calculated to be $\epsilon_{g} \approx 2.5 \times$ $10^{-3} \mathrm{~nm} /$ minute. If, in addition, all 1324 dipole correctors are used the growth rate may be as low as $\epsilon_{g} \approx 9.0 \times$ $10^{-4} \mathrm{~nm} /$ minute for the basic design strategy. As will be shown later, such a large number of correctors can however not be used, since it makes the feedbacks extremely sensitive to certain corrector imperfections. By using Eqs. (51) and (52), the optimal achievable emittance growth rate, for $1324 \mathrm{BPMs}$, was determined to be $\epsilon_{g} \approx 1.75 \times$ $10^{-4} \mathrm{~nm} /$ minute, i.e. $\epsilon_{g} \approx 0.01 \mathrm{~nm} /$ hour. In the following section, the construction of a feedback system with such performance will be described.

\section{A. Construction of optimized feedbacks}

While the construction of a basic feedback system was described in detail in Sec. III C2, it has in the discussion above not been explained how a feedback system with a particular optimized $\mathbf{R}_{c B}$ can be constructed. The optimized matrix $\mathbf{R}_{c B}$ obviously describes how a linear system disturbed by a certain ground motion spectrum should optimally change the coordinates of the beam for a certain BPM reading. The corresponding matrix $\mathbf{R}_{B B}$, describing the desired BPM change, is obviously $-\mathbf{I}$, i.e., the negative identity matrix. The available correctors can, as was seen in Sec. III C 2, be described by the matrices $\mathbf{R}_{c d}$ and $\mathbf{R}_{B d}$. New correctors that are optimized for the optics of the machine and the particular ground motion spectrum of the site may be designed by constructing linear combinations such that

$$
\left(\begin{array}{l}
\mathbf{R}_{c d} \\
\mathbf{R}_{B d}
\end{array}\right) \mathbf{X}=\left(\begin{array}{c}
-\mathbf{R}_{c g} \mathbf{R}_{B g}^{-1} \\
-\mathbf{I}
\end{array}\right) .
$$

This equation system has 1324 unknown (if all correctors are used) and 1618 (294 coordinates + 1324 BPM readings) equations, and an exact solution will most likely not exist. If the equation system is solved in the least-square sense, the feedback performance will not be the desired. The reason is that the feedback correction is based on information from all 1324 BPMs, and the feedbacks will try to steer the beam through the center of all BPMs. If only an approximate solution to Eq. (56) exists, the beam trajectory part will not be exactly $-\mathbf{I}$, and after a first correction step the BPM readings will not be exactly zero. The system will during the next pulse record nonzero BPM readings and will continue correcting the original imperfection. In this way, the BPM readings will finally all become zero, and since there is (in theory) a unique way of using the 1324 dipole correctors to do this, the solution will be identical to what would be obtained using basic feedback design (without optimized coordinate correction).

One way to solve this problem is to relax the equation system by not considering all information obtained from 
the BPMs. In that way, an equation system with a smaller number of equations than unknown may be obtained. This can be done either by removing the least important BPMs, or, as will done here, by transforming the BPM readings to a more convenient space and considering only the most important directions of the new space. In order to construct the new BPM space, a slightly different way of determining the optimal $\mathbf{R}_{c B}$ is introduced. Through the singular value decomposition of $\mathbf{R}_{B g}$ [Eq. (54)], three new matrices $(\mathbf{U}, \mathbf{W}$, and $\mathbf{V})$ were introduced. The orthogonal matrices $\mathbf{U}$ and $\mathbf{V}$ have $1324 \times 1324$ and $1618 \times 1618$ elements, respectively. The matrix $\mathbf{W}$ is obviously $1324 \times 1618$ with nonzero elements (singular values) only in the diagonal. This means that the last 294 columns of $\mathbf{V}$ belong to the null space of $\mathbf{R}_{B g}$ and from Eq. (25) it is clear that the components of $\mathbf{r}$ along these directions cannot be determined by looking at the BPM readings. In addition, components of $\mathbf{r}$ along the columns of $\mathbf{V}$ corresponding to low singular values have little effect on the BPM readings. If Eq. (25) is solved for $\mathbf{r}$ :

$$
\mathbf{r}=\mathbf{V} \mathbf{W}^{-1} \mathbf{U}^{T} \mathbf{b},
$$

the best possible prediction of $\mathbf{r}$ is obtained. This expression, together with Eq. (24), leads to the best achievable prediction of $\mathbf{y}$ :

$$
\mathbf{y}=\mathbf{R}_{c g} \mathbf{V} \mathbf{W}^{-1} \mathbf{U}^{T} \mathbf{b},
$$

which once again shows that the optimal response of the feedback system is described by Eq. (55).

Singular value decomposition of the optimal $\mathbf{R}_{c B}$ shows which BPM patterns have the strongest influence on emittance:

$$
\mathbf{R}_{c B}=\mathbf{U}_{c B} \mathbf{W}_{c B} \mathbf{V}_{c B}^{T} .
$$

Obviously, the first column of $\mathbf{V}_{c B}$ (corresponding to the highest singular value of $\mathbf{R}_{c B}$ ) is the "most important" BPM pattern, leading to a coordinate change along the first column of $\mathbf{U}_{c B}$ with a magnitude determined by the singular value.

From this point onwards, the BPM readings will be studied in the space of the most important BPM patterns. The transformation to this space is carried out simply as

$$
\mathbf{b}^{\prime}=\mathbf{V}_{c B}^{T} \mathbf{b}
$$

where a primed vector will be used to denote the BPM readings in the new space. Similarly, a response matrix describing the response to BPM readings in the new space is

$$
\mathbf{R}_{c B^{\prime}}=\mathbf{U}_{c B} \mathbf{W}_{c B} .
$$

If one component of $\mathbf{b}^{\prime}$ is 1 and all others are 0 , the optimal coordinate change is determined by the corresponding column of $\mathbf{U}_{c B}$ (direction) and singular value of $\mathbf{W}_{c B}$ (magnitude). Under the assumption that the machine is in

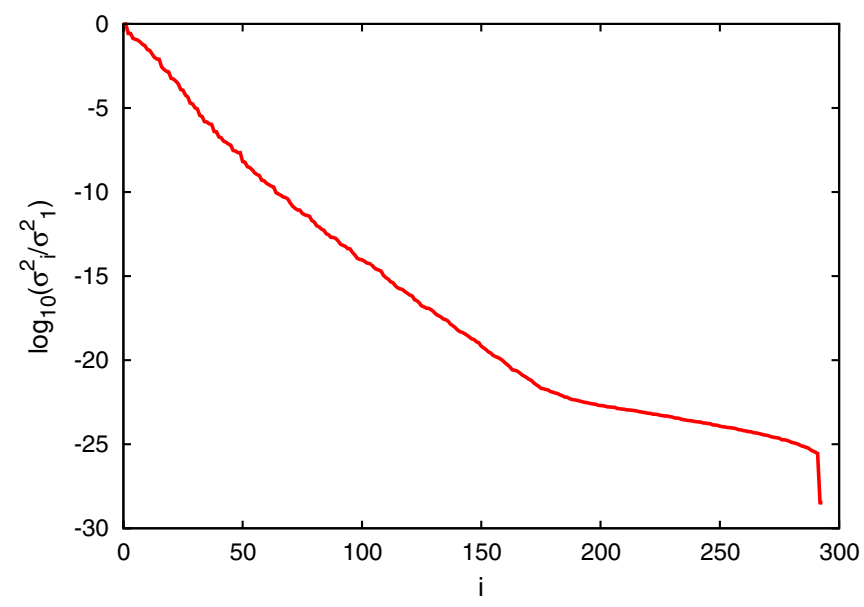

FIG. 5. (Color) Squared singular values of $\mathbf{R}_{c B^{\prime}}$ relative to $\sigma_{1}^{2}$. The plotted value is proportional to the emittance change corresponding to a certain BPM pattern. Only 294 BPM patterns yield a nonzero value. All other patterns belong to the null space of $\mathbf{R}_{c B^{\prime}}$.

a regime where the second-order emittance growth approximation is accurate, the emittance reduction achieved by the optimal coordinate change equals the singular value squared. By plotting the squared singular values of $\mathbf{R}_{c B^{\prime}}$, it is clear that most BPM patterns have almost no importance for emittance preservation, see Fig. 5. The optimal performance of a feedback system using the BPM pattern components along a limited number of directions was calculated by determining $\mathbf{R}_{c B}$ for the $n$ most important BPM patterns:

$$
\mathbf{R}_{c B^{\prime}}^{\prime}=\mathbf{U}_{c B} \mathbf{W}_{c B}^{\prime} .
$$

Here, $\mathbf{W}_{c B}^{\prime}$ contains only the first $n$ columns of $\mathbf{W}_{c B}^{\prime}$. The lowest achievable emittance growth rate may then be calculated using Eq. (51). In Fig. 6, it is shown that no more than 25-30 BPM patterns are required to achieve nearly optimal performance. The 30 first BPM patterns were considered to contain sufficient information for efficient emittance preservation (with an emittance growth rate of $\epsilon_{g} \approx 1.75 \times 10^{-4} \mathrm{~nm} /$ minute). By neglecting all other directions in the new BPM space, a relaxed [compared to Eq. (56)] equation system is obtained. The new equation system is

$$
\left(\begin{array}{c}
\mathbf{R}_{c d} \\
\mathbf{R}_{B^{\prime} d}
\end{array}\right) \mathbf{X}=\left(\begin{array}{c}
-\mathbf{R}_{c g} \mathbf{R}_{B^{\prime} g}^{-1} \\
-\mathbf{I}
\end{array}\right)
$$

where $\mathbf{R}_{B^{\prime} d}$ contains the 30 first rows of $\mathbf{V}_{c B}^{T} \mathbf{R}_{B d}$. Similarly $\mathbf{R}_{B^{\prime} g}$ contains the 30 first rows of $\mathbf{V}_{c B}^{T} \mathbf{R}_{B g}$.

By using all 1324 dipole correctors, the relaxed equation system could be solved exactly both for the coordinate and for the BPM part of the equations. Consequently, the problem discussed above was avoided. Simulations showed that the emittance growth rate is as low as analytically predicted, see Fig. 7. 


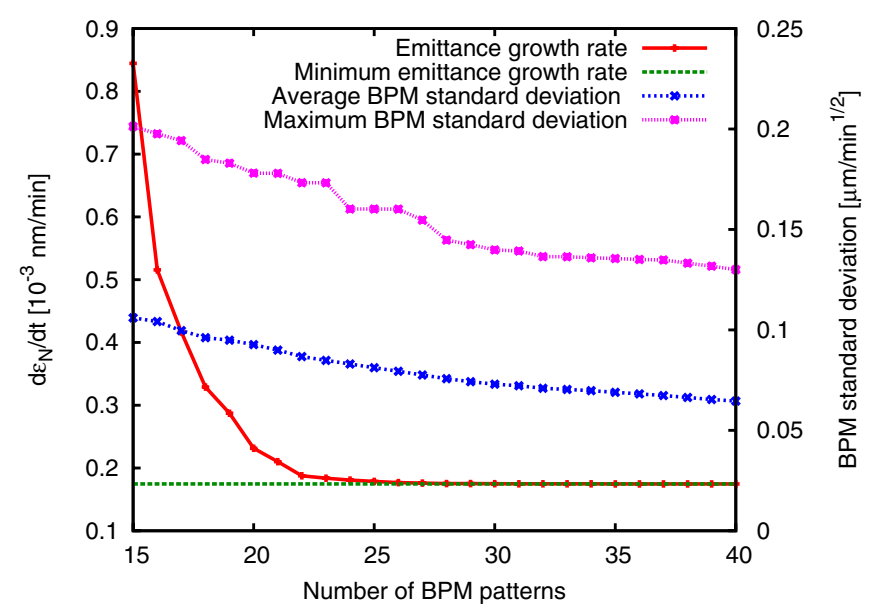

FIG. 6. (Color) Minimum achievable emittance growth rate for different numbers of BPM patterns. No more than the 25-30 most important patterns have to be considered to achieve almost the same (optimal) performance as when all BPM information is taken into account. The figure also shows how well the trajectory can be controlled with information from a limited number of BPM patterns.

As previously mentioned, using a large number of dipole correctors will make the feedback system sensitive to corrector errors. It was therefore studied how far the number of correctors could be reduced while still keeping the optimal feedback performance. One by one, the correctors that were most useful for solving Eq. (63) were selected. Observe that, if the BPM part of the equation is not solved exactly, the performance will not be the desired. The equation system was therefore weighted to make sure that the BPM part was (nearly) exactly reconstructed. It was seen that 50-70 correctors is sufficient to obtain almost optimal performance, see Fig. 8. Apparently, an

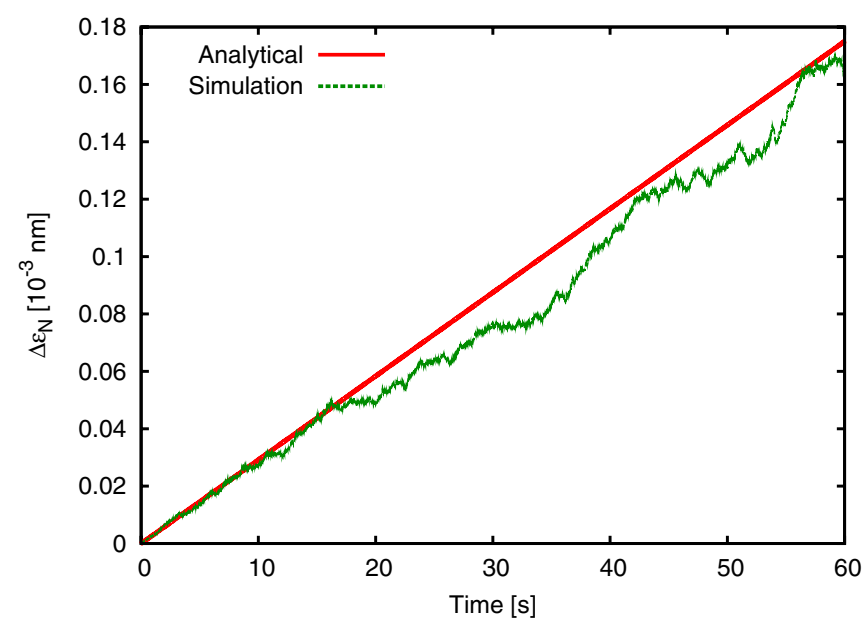

FIG. 7. (Color) Average emittance growth during $1 \mathrm{~min}$ (9000 pulses) for 50 random seeds. Only ground motion and feedbacks with ideal BPMs were included. The simulations agree well with the analytical prediction.

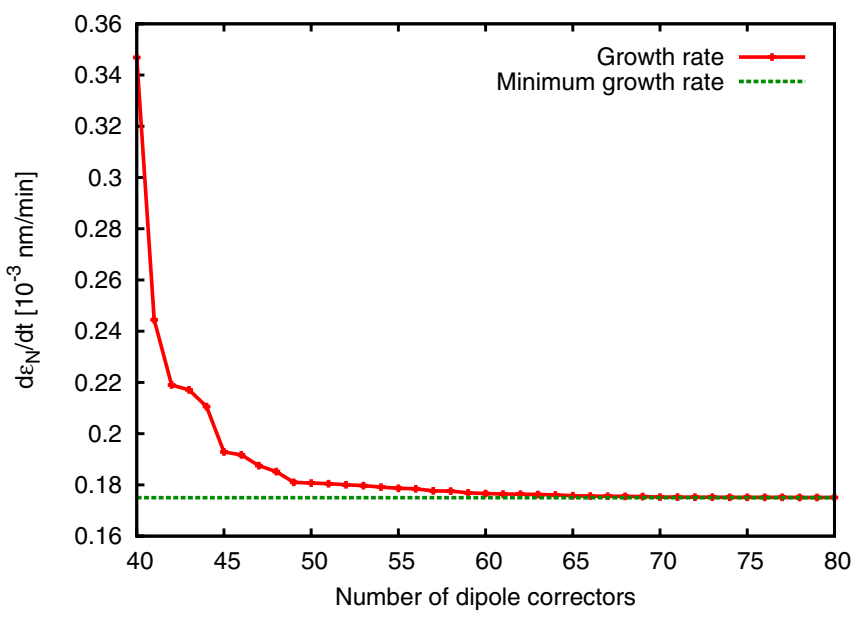

FIG. 8. (Color) Emittance growth rate for an optimized feedback system constructed using a variable number of dipole correctors. Using as few as 60 correctors, a performance within $1 \%$ of what is optimal can be obtained. For 70 correctors, the growth rate is approximately $0.14 \%$ higher than the optimal. Note that the corrector that is most useful for solving Eq. (63) is not necessarily the most efficient for feedback. This is, e.g., seen between corrector 42 and 45 where the correctors are selected in the wrong order. As a consequence, it could be possible to use even a few correctors less if a more advanced selection algorithm is used.

optimized feedback system, which preserves emittance much better than the basic feedback system described in Sec. III C 2, can be constructed using as few as 60 correctors. In terms of emittance growth rate, the new feedback system is approximately 14 times more efficient than a basic trajectory feedback system based on all 1324 BPMs and 80 equally spaced dipole correctors.

If the two feedback systems are instead compared in terms of their ability to steer the beam to the reference trajectory, the basic system, however, works much better. The standard deviation of the final BPM readings, for a large number of random machines after $1 \mathrm{~min}$ of ground motion and feedback, was calculated for each BPM of the main linac. This was simply done by calculating the norm of each row of

$$
\mathbf{R}_{B g}+\mathbf{R}_{B B^{\prime}} \mathbf{R}_{B^{\prime} g}
$$

and multiplying by $(60 \times 150)^{1 / 2} \times \sigma_{\mathrm{gm}}$. In Eq. (64), the response matrix $\mathbf{R}_{B B^{\prime}}$ is used to denote the feedback response of BPM readings to a change in a BPM pattern. This way of calculating an expected trajectory deviation is similar to the way expected emittance growth was determined in Sec. III C 3. Observe, however, that the trajectory deviations grow with the square root of time, as opposed to linearly with time for emittance growth. In Fig. 9, it is clearly shown that the basic feedback system reduces trajectory deviations efficiently. This predicted performance was in nearly exact agreement with simulations of 50 


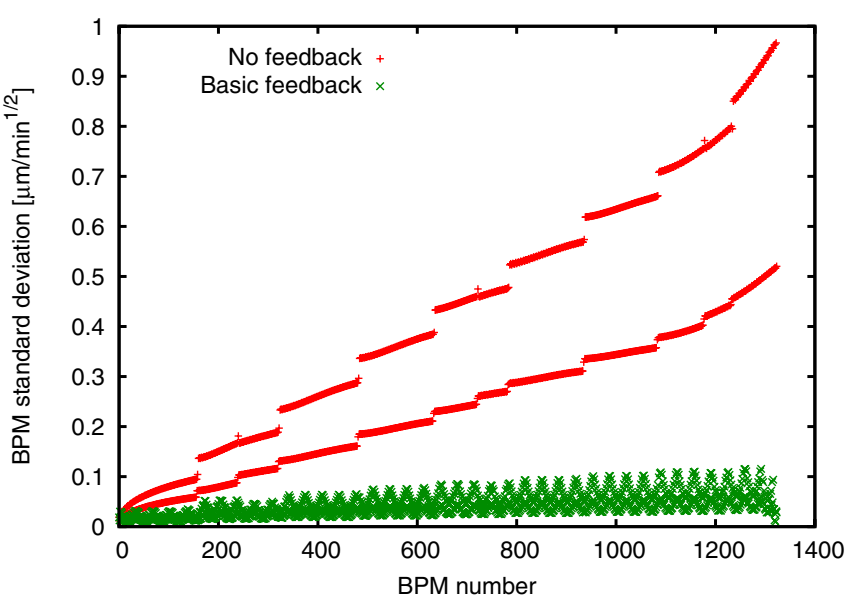

FIG. 9. (Color) Theoretical standard deviation of BPM readings for a large number of seeds. Without feedback the trajectory deviation at the end of the linac is of the order of a $\mu \mathrm{m}$ after $1 \mathrm{~min}$. The basic feedback system based on 1324 BPMs and 80 dipole corrector efficiently reduces the deviations.

random seeds. For the feedback system designed for optimal emittance performance, the trajectory deviations are instead deteriorated as compared to not using feedback at all. For an optimized feedback system constructed using 60 correctors, the maximum trajectory deviations were seen to be roughly $16 \mu \mathrm{m}$ after $1 \mathrm{~min}$. For a larger number of correctors, the performance was even worse. The reason for this deterioration is that Eq. (63) was solved without considering how the BPM components along the 1294 least important (for emittance preservation) directions were changed. In order to avoid this problem, the construction of the feedbacks has to be slightly modified. The modification was in this case carried out by adding equations such that the BPM pattern components along the 1294 least important directions were changed according to the best achievable prediction (in the same way as for the coordinate correction):

$$
\left(\begin{array}{l}
\mathbf{R}_{c d} \\
\mathbf{R}_{B^{\prime} d} \\
\mathbf{R}_{B^{\prime \prime} d}
\end{array}\right) \mathbf{X}=\left(\begin{array}{c}
-\mathbf{R}_{c g} \mathbf{R}_{B^{\prime} g}^{-1} \\
-\mathbf{I} \\
-\mathbf{R}_{B^{\prime \prime} g} \mathbf{R}_{B^{\prime} g}^{-1}
\end{array}\right),
$$

where $\mathbf{R}_{B^{\prime \prime} d}$ and $\mathbf{R}_{B^{\prime \prime} g}$ contain the last 1294 rows of $\mathbf{V}_{c B}^{T} \mathbf{R}_{B d}$ and $\mathbf{V}_{c B}^{T} \mathbf{R}_{B g}$, respectively. Observe that the only information that will be used for steering will still be the 30 BPM components along the most important directions. Each of the 30 columns of the matrix on the right-hand side represents the desired feedback response to a unit change along the corresponding BPM pattern. In Fig. 6, it was shown that by using 30 BPM patterns the maximum BPM standard deviation growth rate can, in theory, be reduced to approximately $0.14 \mu \mathrm{m} / \mathrm{min}^{1 / 2}$. The efficient reduction of the trajectory deviations is also shown in Fig. 10.

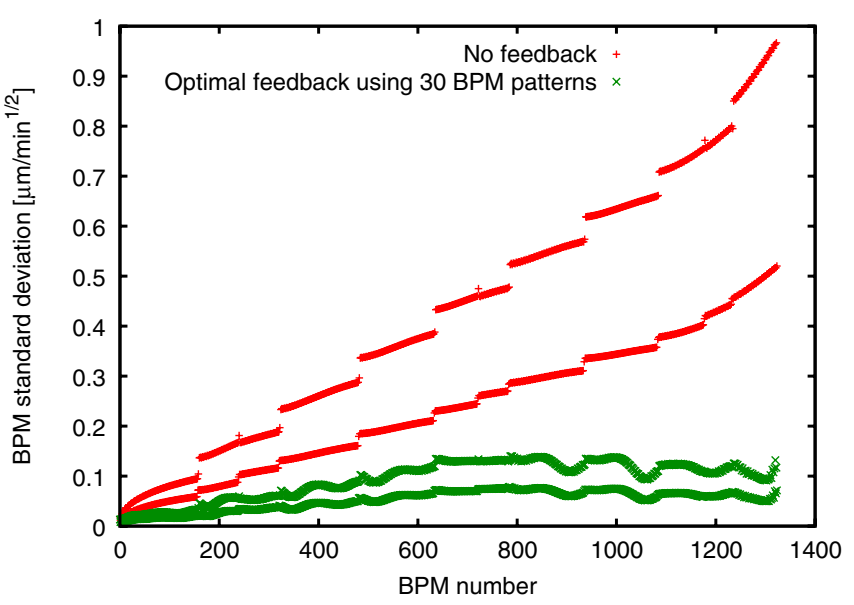

FIG. 10. (Color) Theoretical standard deviation of BPM readings for a large number of seeds. A feedback system based on 30 BPM patterns could ideally [if Eq. (65) could be solved exactly] reduce deviations efficiently.

It should be mentioned that Eq. (65) is a simplification of the equation actually solved. As mentioned above, the part of the equation system containing the most important BPM information should be solved exactly. The part containing the beam coordinates should also be solved with high precision, while the additional BPM readings are considered less important. A weight was therefore given to each of the three parts to reflect the required precision of the solution. Unit weight was given to the coordinate part, a weight of $\approx 10^{3}$ to the 30 most important BPM patterns, and finally a weight of $\approx 10^{-3}$ to the remaining $1294 \mathrm{BPM}$ patterns. The low weight on the 1294 least important BPM patterns means that the ideal trajectory performance shown in Fig. 10 cannot be obtained. Additional weights were also given to each row of the part with the important BPM patterns. This was done in order to reflect the fact that an error on the row corresponding to the most important pattern would cause the largest deviation from optimal performance (in terms of emittance).

After selecting 80 useful correctors (as few as 60-70 also seemed to yield reasonably good results), the equation system was solved and the new feedbacks were tested. The emittance performance was seen to be similar (growth rate increased with a few percent) to what was obtained before including the less important BPM directions. In addition, the new feedback system corrects the trajectory quite well. By tweaking with the weights, the performance could be adjusted such that the emittance growth rate was $\epsilon_{g} \approx$ $1.81 \times 10^{-4} \mathrm{~nm} / \mathrm{min}$ and the growth rate of the maximum trajectory deviation was $0.69 \mu \mathrm{m} / \mathrm{min}^{1 / 2}$. If trajectory deviations of $20 \mu \mathrm{m}$ are considered acceptable, the system can run for 840 minutes before this limit is reached. Similarly, if $0.15 \mathrm{~nm}$ emittance growth is acceptable (ground motion would then contribute with approximately the same amount of emittance growth as jitter cf. Fig. 4), 


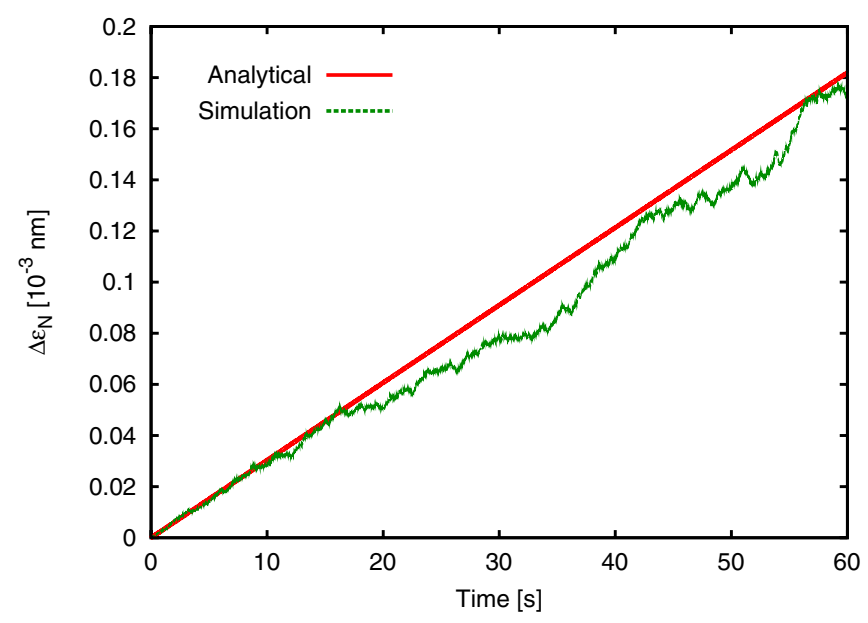

FIG. 11. (Color) Average emittance growth during 1 min (9000 pulses) for 50 random seeds. Only ground motion and feedbacks with ideal BPMs were included. The optimized feedback system used 30 BPM patterns (all 1324 BPMs) and was constructed using 80 correctors. The corresponding trajectory control performance is shown in Fig. 12.

the feedback system can run for 830 minutes. This means that the optimized feedbacks could possibly control the ground motion for almost 14 hours. The analytical results and results of simulations of 50 machines are shown in Figs. 11 and 12.

Since the optimized system relies on knowledge about the optics of the machine and different response matrices, it is of great interest to understand to what extent imperfect system knowledge affects the emittance growth performance. As shown in the following section, slight modifications of the feedback design may be necessary to avoid the need for very accurate knowledge of the system.

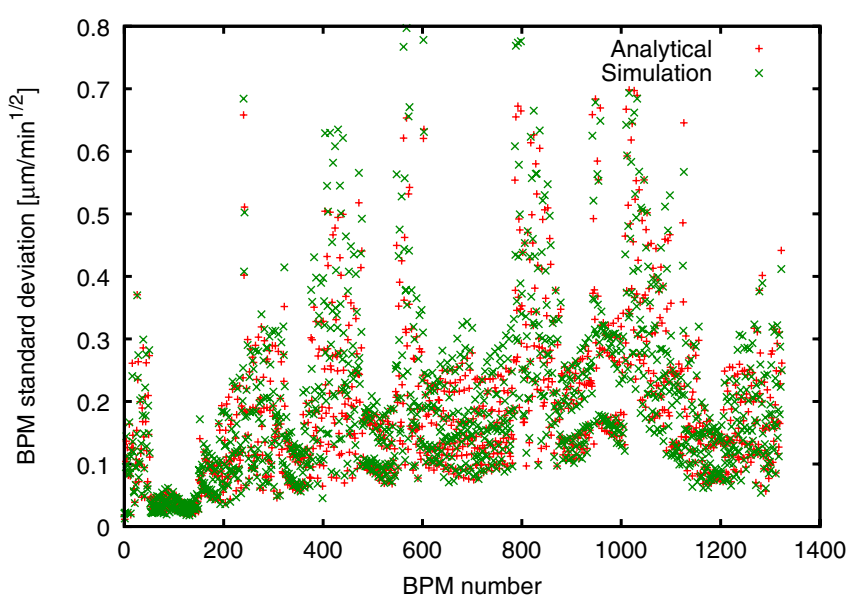

FIG. 12. (Color) Standard deviation of BPM readings for a large number of seeds. A feedback system based on 30 BPM patterns and 80 correctors is seen to control trajectory deviations reasonably well. Simulations and analytical results agree well.

\section{Imperfect system knowledge}

The new optimized feedback system apparently performs much better than basic feedbacks in terms of emittance growth rate. The design of a feedback system relies on good knowledge of the dipole corrector response matrices. In case of the optimized feedbacks, knowledge of the response to girder movements (caused by ground motion) is also necessary. In order to make sure that the requirements on system knowledge are not too demanding, a few simulations were carried out under the assumption that the optics of the machine were not exactly known. For simplicity, only the strength of the quadrupoles was varied. Initially, two different feedback systems designed for the ideal optics were tested. The first was a basic feedback system based on 1324 BPMs and 80 dipole correctors (designed in the same way as the system described in Sec. IIIC 2). The second was the optimized feedback system constructed in Sec. IVA. This system is based on the use of the 30 most important BPM patterns (all 1324 BPMs) and 80 selected dipole correctors.

Both feedback designs were tested for four different optics: the ideal optics and optics with relative quadrupole strength errors with $\sigma=0.01 \%, \sigma=0.1 \%$, and $\sigma=1 \%$, respectively. The tests were carried out using 20 random machines for each alternative. The basic feedback design did not seem to be strongly affected by the $0.01 \%$ and $0.1 \%$ errors. For the $1 \%$ error, however, quite strong emittance fluctuations were visible, see Fig. 13. For the optimized feedback system, the $1 \%$ error led to an enormous increase in emittance growth rate (more than a factor 100). The $0.1 \%$ error caused a factor 2 increase, while in the $0.01 \%$ case little change was seen as compared to the ideal optics $\left(\epsilon_{g} \approx 0.18 \times 10^{-3} \mathrm{~nm}\right)$. In order to reduce the sensitivity, the solution of the coordinate part of Eq. (65) was relaxed by readjusting the weights. With lower relative weight on

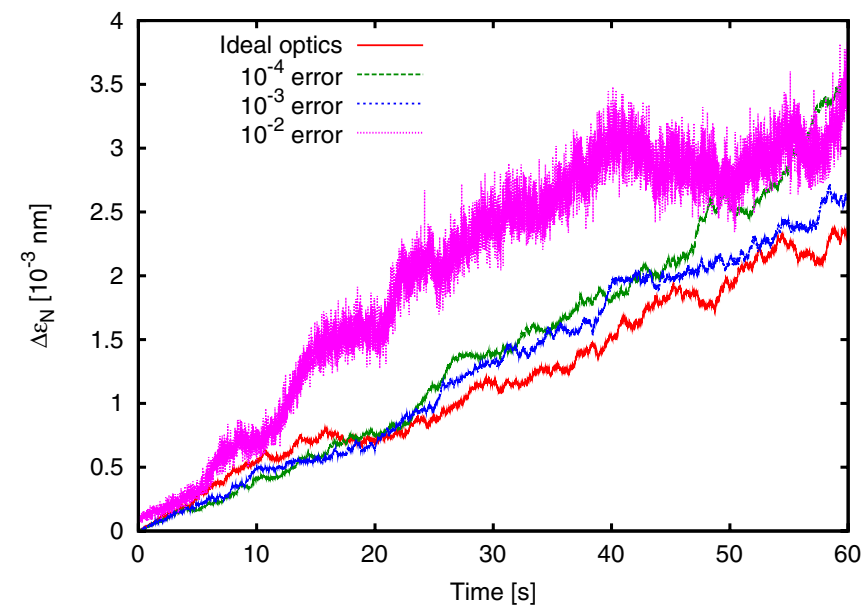

FIG. 13. (Color) Emittance growth vs time for a basic feedback system using 1324 BPMs and 80 correctors. The feedback performance is seen not to be strongly affected by imperfect knowledge of the machine optics. 


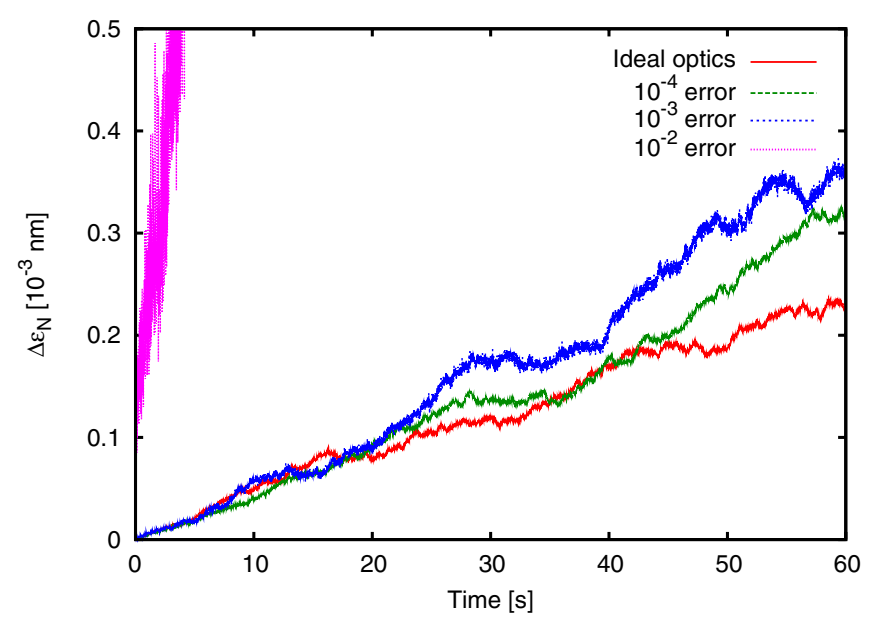

FIG. 14. (Color) Emittance growth vs time for an optimized feedback system using 30 BPM patterns (1324 BPMs) and 80 correctors with an expected emittance growth rate of roughly $0.28 \times 10^{-3} \mathrm{~nm} / \mathrm{min}$ (due to the few random seeds used, even the ideal optics clearly deviate from this value). Apart from the $1 \%$ error (with a growth rate of $\approx 7 \times 10^{-3} \mathrm{~nm} / \mathrm{min}$ ), the system is reasonably tolerant to the faulty system knowledge.

the coordinate part, two new optimized feedback systems were constructed: one with an emittance growth rate of $\epsilon_{g} \approx 0.28 \times 10^{-3} \mathrm{~nm} / \mathrm{min}$ and one with an emittance growth rate of $\epsilon_{g} \approx 0.40 \times 10^{-3} \mathrm{~nm} / \mathrm{min}$. The slightly less "emittance-optimized" feedback design turned out to be quite tolerant to the imperfect system knowledge, see Figs. 14 and 15.

By testing a few more optimized feedback systems, it was seen that the best achievable emittance growth rate for an optics error of $0.1 \%$ is approximately $0.3 \times 10^{-3} \mathrm{~nm}$.

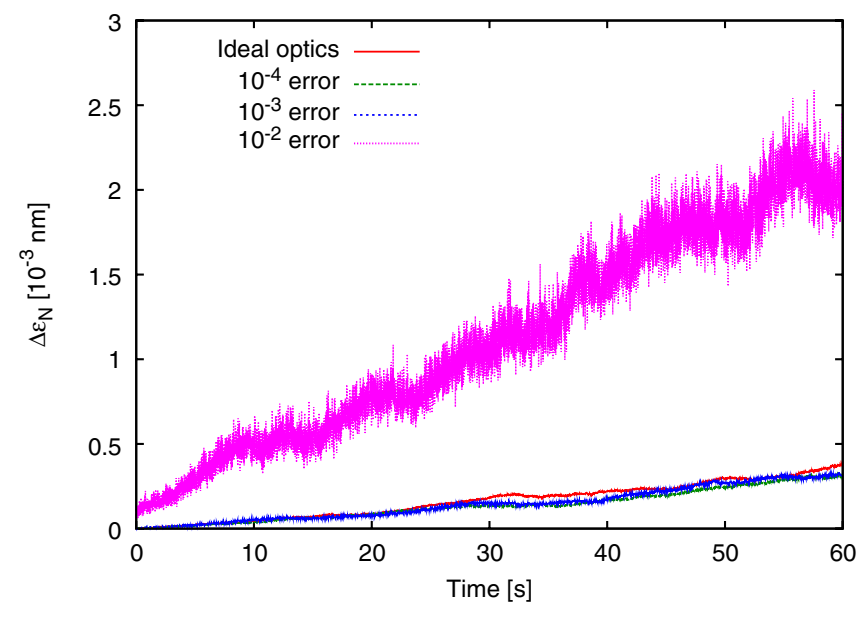

FIG. 15. (Color) Emittance growth vs time for a basic feedback system using 30 BPM patterns (1324 BPMs) and 80 correctors and with an expected emittance growth rate of $\approx 0.40 \times$ $10^{-3} \mathrm{~nm} / \mathrm{min}$. Apart from the $1 \%$ error, the feedback performance is not strongly affected by imperfect knowledge of the machine optics.
Apparently, the optimized feedback design relies more on good system knowledge than the basic feedback design. However, optimized feedbacks can be designed to be nearly as tolerant (for errors up to $0.1 \%$ ) and still be almost 10 times more efficient (in terms of emittance growth rate) than the basic feedbacks. Observe that, for the $1 \%$ error, the deteriorated feedback performance may not be the most severe problem. In that case, it is found that there is an emittance growth of $\Delta \epsilon_{N} \approx 1.6 \mathrm{~nm}$ along the linac even for a perfectly aligned linac.

\section{Gain optimization}

It has been shown that an optimized feedback system based on the use of 80 correctors can be used to reduce the emittance growth rate very efficiently. It is, however, also important to make sure that the constant (independent of time) emittance growth terms of Eq. (50) are kept at a minimum. While the direct emittance growth caused by jitter [first three terms of Eq. (50)] is independent of the feedback, there are a number of terms contributing to emittance growth which depend on the gain $g$. These terms describe the indirect effect of jitter and feedback, the effect of finite BPM resolution, and the effect of slow feedback correction (two last terms).

For the optimized feedback system, the scaling factors for indirect jitter-induced emittance growth were calculated to be

$$
\begin{gathered}
e_{f b, q} \approx 8.72 \times 10^{4} \mathrm{~nm} / \mu \mathrm{m}^{2}, \\
e_{f b, s} \approx 0.38 \mathrm{~nm} / \mu \mathrm{m}^{2}, \\
e_{f b, b} \approx 5.15 \mathrm{~nm} / \mu \mathrm{m}^{2} .
\end{gathered}
$$

These values are similar to what was obtained for the basic feedback setup [see the paragraph following Eq. (37)]. For the imperfections of Table II, the total indirect emittance growth is seen to be slightly smaller for the optimized feedback system, but the difference is very small and completely negligible compared to the direct emittance growth caused by jitter $(0.138 \mathrm{~nm})$.

The scaling factor for feedback BPM noise was then determined according to Eq. (35). Because of the fact that all BPMs are used, the sensitivity to BPM noise is clearly reduced. The scaling factor for the optimized feedback system based on 30 BPM patterns (1324 BPMs) is

$$
e_{f b} \approx 4.53 \mathrm{~nm} / \mu \mathrm{m}^{2} .
$$

In order to minimize these gain-dependent emittance contributions, the optimal gain was determined in the same way as for the basic feedback system in Sec. III C 2. The optimal value was seen to be $g \approx 0.093$. For this gain, the emittance contribution from gaindependent terms is approximately $0.019 \mathrm{~nm}$, leading to a 
total constant emittance growth (including direct jitter effects) of $\Delta \epsilon_{N} \approx 0.138+0.019 \mathrm{~nm}=0.157 \mathrm{~nm}$.

In principle, a more general approach than using a single gain parameter would be to use different gains for different BPM patterns. Since each column of $\mathbf{R}_{c B^{\prime}}$ contains the optimal response to a unit component along the corresponding BPM pattern, different fractional corrections (gains) would lead to a modification of Eqs. (31) and (32):

$$
\begin{aligned}
\Delta \mathbf{y} & =\mathbf{R}_{c B^{\prime}} \mathbf{G} \mathbf{b}^{\prime}, \\
\Delta \mathbf{b} & =\mathbf{R}_{B B^{\prime}} \mathbf{G} \mathbf{b}^{\prime},
\end{aligned}
$$

where the "gain matrix" $\mathbf{G}$ is a diagonal matrix containing the gain parameter of each BPM pattern:

$$
\mathbf{G}=\left(\begin{array}{cccc}
g_{1} & 0 & \cdots & 0 \\
0 & g_{2} & \cdots & 0 \\
\vdots & \vdots & \ddots & \vdots \\
0 & 0 & \cdots & g_{30}
\end{array}\right) .
$$

The motivation for using different gains can be indicated by a plot showing the expected BPM pattern component caused by different imperfections, see Fig. 16. For BPM noise, the rms reading of each BPM is $\sigma_{\mathrm{BPM}}$. Since the transformation to the new BPM basis is orthonormal ( $\mathbf{V}_{c B}$ is orthogonal), the same rms value is expected for each BPM pattern. For the other imperfections, the standard deviation varies strongly for different BPM patterns. While ground motion is ideally corrected with gain $g=$ 1 (assuming perfect BPMs), the other imperfections, which vary from pulse to pulse, are less severe for a low gain.

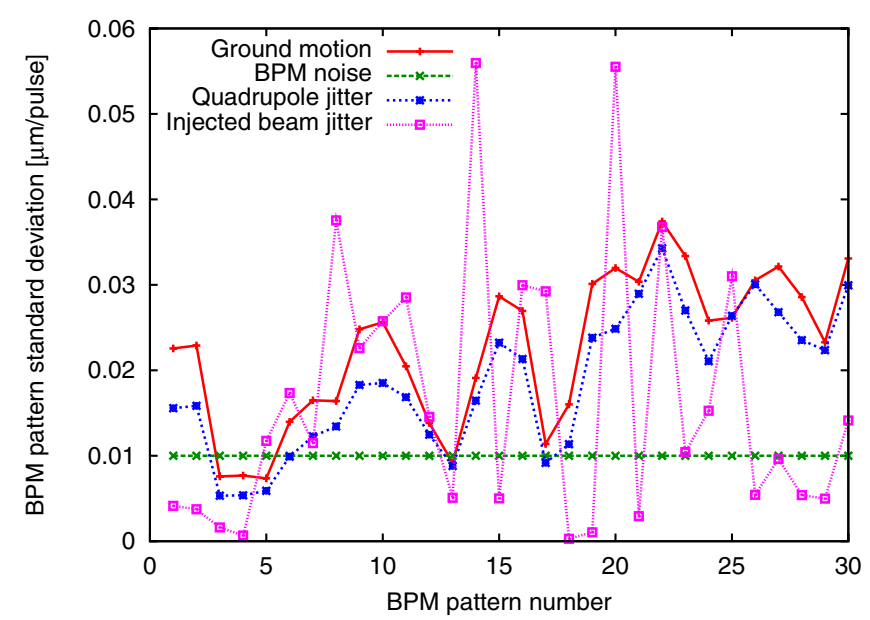

FIG. 16. (Color) Theoretical standard deviation of BPM pattern components for different imperfections after one pulse. While ground motion causes a lasting deviation, the other imperfections cause deviations that are uncorrelated from one pulse to another. They have therefore been reduced by a factor 10 to reflect the effect of a gain $g=0.1$. Note the similarity between the results for ground motion and quadrupole jitter. This is a consequence of the fact that ground motion effects are mainly caused by displaced quadrupoles.
Since the deviations along the first two patterns are largest for ground motion, a higher gain can be used for these patterns than for, e.g., patterns 5, 8, and 14 (for which at least one of the other imperfections are more important). As shown below, these simple considerations are indeed confirmed by a more detailed study. Observe that, if Fig. 16 is extended to include all BPM patterns, it is clearly seen that BPM noise dominates completely for all but the first $\approx 50$ patterns.

If a gain matrix $\mathbf{G}$, with diagonal elements $g_{j}$, is used instead of a single gain parameter $g$, the emittance growth caused by ground motion and feedback [cf. Eqs. (44) and (45)], is modified as

$$
\begin{aligned}
\left\langle\Delta \epsilon_{N}\right\rangle \approx & \sigma_{\mathrm{gm}}^{2} \sum_{i=0}^{P-1}\left\{\left\|\mathbf{R}_{S}\right\|_{F}^{2}-2 \operatorname{Tr}\left(\mathbf{R}_{S}^{T} \mathbf{R}_{c B^{\prime}} \tilde{\mathbf{G}}^{i} \mathbf{R}_{B^{\prime} g}\right)\right. \\
& \left.+\operatorname{Tr}\left[\mathbf{R}_{B^{\prime} g}^{T}\left(\tilde{\mathbf{G}}^{i}\right)^{T} \mathbf{R}_{c B^{\prime}}^{T} \mathbf{R}_{c B^{\prime}} \tilde{\mathbf{G}}^{i} \mathbf{R}_{B^{\prime} g}\right]\right\} \\
= & \sigma_{\mathrm{gm}}^{2} P e_{\mathrm{gm}, 0} \\
& +\sigma_{\mathrm{gm}}^{2} \sum_{i=0}^{P-1}\left[-2 \operatorname{Tr}\left(\tilde{\mathbf{G}}^{i} \mathbf{A}\right)+\operatorname{Tr}\left(\tilde{\mathbf{G}}^{i} \mathbf{B} \tilde{\mathbf{G}}^{i} \mathbf{C}\right)\right],
\end{aligned}
$$

where

$$
\tilde{\mathbf{G}}=\mathbf{I}-\mathbf{G} .
$$

In the second step of Eq. (73), the cyclic property of trace was used and three new matrices $(\mathbf{A}, \mathbf{B}$, and $\mathbf{C})$ were introduced. These matrices are

$$
\begin{gathered}
\mathbf{A}=\mathbf{R}_{B^{\prime} g} \mathbf{R}_{S}^{T} \mathbf{R}_{c B^{\prime}}, \\
\mathbf{B}=\mathbf{R}_{c B^{\prime}}^{T} \mathbf{R}_{c B^{\prime}}, \\
\mathbf{C}=\mathbf{R}_{B^{\prime} g} \mathbf{R}_{B^{\prime} g}^{T} .
\end{gathered}
$$

By denoting the elements of $\mathbf{A}, \mathbf{B}$, and $\mathbf{C}$ by $a_{j k}, b_{j k}$, and $c_{j k}$, respectively, and the diagonal elements of $\tilde{\mathbf{G}}$ by $\tilde{g}_{j}$, Eq. (73) may be written

$$
\begin{aligned}
\left\langle\Delta \epsilon_{N}\right\rangle & \approx \sigma_{\mathrm{gm}}^{2}\left(P e_{\mathrm{gm}, 0}-2 \sum_{i, j} \tilde{g}_{j}^{i} a_{j j}+\sum_{i, j, k} \tilde{g}_{j}^{i} b_{j k} \tilde{g}_{k}^{i} c_{k j}\right) \\
& \approx \sigma_{\mathrm{gm}}^{2}\left(P e_{\mathrm{gm}, 0}-2 \sum_{j} \frac{a_{j j}}{g_{j}}+\sum_{j, k} \frac{z_{j k}}{g_{j}+g_{k}-g_{j} g_{k}}\right),
\end{aligned}
$$

where $z_{j k}=b_{j k} c_{k j}$. The summation index $i$ runs from 0 to $P-1$ [as in Eq. (73)], and $j$ and $k$ run from 1 to 30 (the number of BPM patterns). In the last step of the derivation, the geometric sums over $i$ were evaluated and transient terms removed under the assumption that $P$ is large. It is easily seen that

$$
\sum_{j} a_{j j}=\operatorname{Tr}(\mathbf{A})=e_{\mathrm{gm}, 1}
$$




$$
\sum_{j, k} z_{j k}=\operatorname{Tr}(\mathbf{B C})=e_{\mathrm{gm}, 2}
$$

Consequently, Eq. (78) equals Eq. (45) if $g_{j}=g, \forall j$.

Similar considerations may be applied to derive emittance growth caused by imperfect BPMs using a gain matrix $\mathbf{G}$.

$$
\begin{aligned}
\left\langle\Delta \epsilon_{N}\right\rangle & \approx \sigma_{\mathrm{BPM}}^{2} \sum_{i=0}^{P-1} \operatorname{Tr}\left[\left(\mathbf{G} \tilde{\mathbf{G}}^{i}\right)^{T} \mathbf{R}_{c B^{\prime}}^{T} \mathbf{R}_{c B^{\prime}} \mathbf{G} \tilde{\mathbf{G}}^{i}\right] \\
& =\sigma_{\mathrm{BPM}}^{2} \sum_{i, j} g_{j}^{2} \tilde{g}_{j}^{2 i} b_{j j} \approx \sigma_{\mathrm{BPM}}^{2} \sum_{j} \frac{g_{j}}{2-g_{j}} b_{j j} .
\end{aligned}
$$

Another three terms contributing to emittance growth depend on the gain. These terms are the indirect effects of quadrupole, accelerating structure, and injected beam jitter. For a single gain $g$, the effect of quadrupole jitter is described by the second term of Eq. (37). In case a gain matrix $\mathbf{G}$ is used, this term changes to

$$
\begin{aligned}
\left\langle\Delta \boldsymbol{\epsilon}_{N}\right\rangle & \approx \sigma_{q}^{2} \sum_{i=0}^{P-1} \operatorname{Tr}\left[\left(\mathbf{G} \tilde{\mathbf{G}}^{i}\right)^{T} \mathbf{R}_{c B^{\prime}}^{T} \mathbf{R}_{c B^{\prime}} \mathbf{G} \tilde{\mathbf{G}}^{i} \mathbf{R}_{B^{\prime} q} \mathbf{R}_{B^{\prime} q}^{T}\right] \\
& =\sigma_{q}^{2} \sum_{i, j, k} g_{j} \tilde{g}_{j}^{i} b_{j k} g_{k} \tilde{g}_{k}^{i} c_{k j} \\
& \approx \sigma_{q}^{2} \sum_{j, k} \frac{g_{j} g_{k}}{g_{j}+g_{k}-g_{j} g_{k}} z_{j k} .
\end{aligned}
$$

In a similar fashion, the indirect emittance growth caused by jittering accelerating structures and injected beam jitter may be derived.

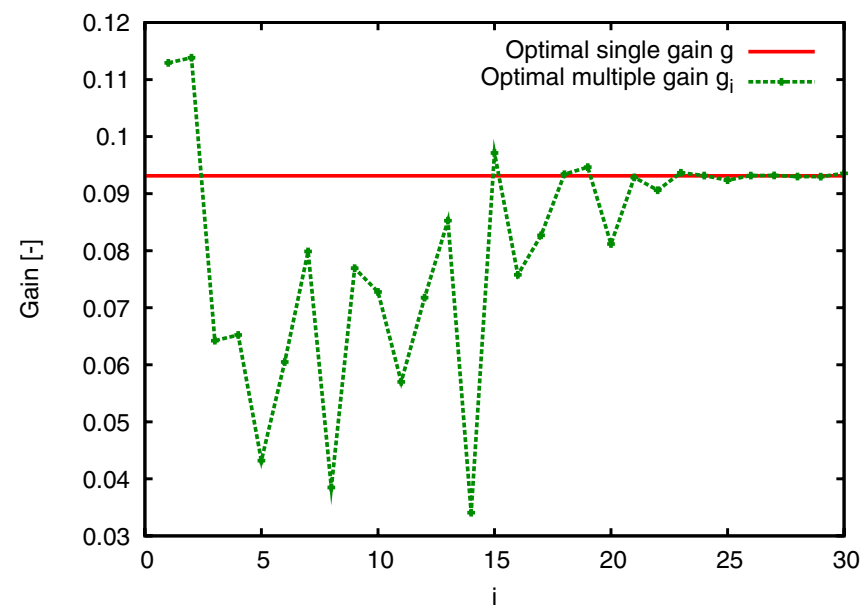

FIG. 17. (Color) Optimal values of a single gain parameter and of multiple gain parameters (for each BPM pattern $i$ ). The optimal single gain $g \approx 0.093$ was used as a starting point for the multiple gain parameters $g_{i}$ during the gradient search. Since the last few BPM patterns correspond to small emittance changes, the corresponding gain parameters were hardly changed by the optimization procedure. Their precise values are of very little importance.

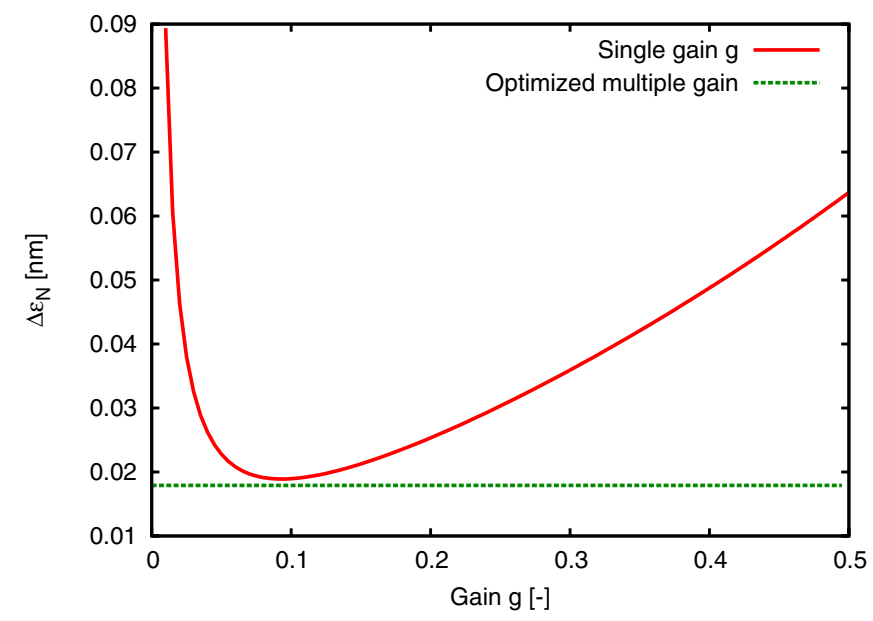

FIG. 18. (Color) Total gain-dependent emittance growth for various values of the single gain parameters and for the optimal multiple gains (see Fig. 17). For the optimal single gain $g \approx$ 0.093 , the emittance growth is $\Delta \epsilon_{N} \approx 0.019 \mathrm{~nm}$ compared to $\Delta \epsilon_{N} \approx 0.018 \mathrm{~nm}$ for optimized multiple gain parameters.

The optimal individual gain parameters $g_{j}$ are obtained by minimizing the sum of the gain-dependent terms derived in Eqs. (78), (81), and (82) (plus corresponding terms for accelerating structure and injected beam jitter). The optimization was in this case carried out using a gradient search method. The optimal gains for the feedback system constructed in Sec. IVA, with imperfections according to Table II, are shown in Fig. 17. As shown in Fig. 18, the emittance decrease obtained by optimizing individual gain parameters is, however, very small (in particular compared to the direct emittance growth $\Delta \epsilon_{N} \approx 0.138 \mathrm{~nm}$ caused by jitter).

In theory, this more general strategy of gain optimization permits a better optimum than for a single gain parameter. In this case, however, little improvement was obtained. The reason could be that the emittance growth is already low, or that the quadrupole jitter, which cause similar BPM pattern deviations as ground motion, limits the gain in all cases where a high gain could otherwise have been used (see e.g. pattern $1,2,15,19)$. In addition, the feedback performance is to a large extent determined by the gain parameters of a few of the most important patterns. In effect, the optimization problem only has a few degrees of freedom rather than 30 .

In order to confirm the predicted emittance growth, 50 machines were simulated during 1 min taking all imperfections into account. The results are in perfect agreement with the theoretical predictions, see Fig. 19.

\section{Further imperfections}

A few additional imperfections were studied to make sure that the optimized feedbacks will perform well even under nonideal conditions. One of these imperfections, namely, dipole corrector step size errors, has already 


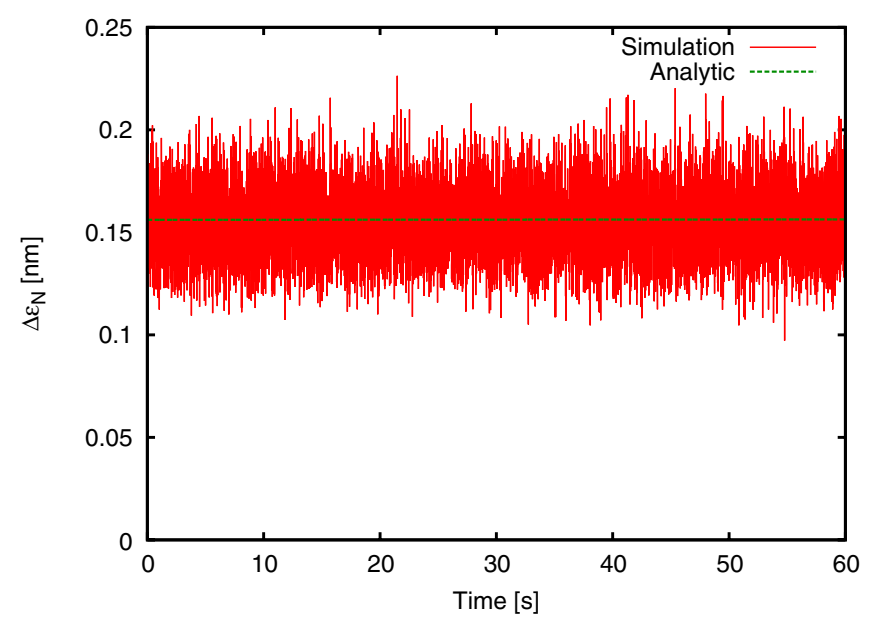

FIG. 19. (Color) Emittance growth along the CLIC main linac during $1 \mathrm{~min}$ of operation. The analytic results are seen to be in perfect agreement with simulations. Compared to the constant emittance growth of $\Delta \epsilon_{N} \approx 0.155 \mathrm{~nm}$, the increase caused by ground motion is not visible after only $1 \mathrm{~min}$.

been mentioned as a reason to use a limited number of correctors. The correctors were simulated as displacements of quadrupoles, and the effect of a $10 \mathrm{~nm}$ minimum step size was investigated. In other words, the quadrupole positions could only assume discrete values with a spacing of $10 \mathrm{~nm}$. Simulations of 20 random machines showed that the step size error increased the emittance growth by $\approx 0.067 \mathrm{~nm}$ for the basic feedback system and by $\approx 0.037 \mathrm{~nm}$ for the optimized feedbacks described above. This is in perfect agreement with theoretical predictions of the effect that jitter of the 80 selected quadrupoles (correctors) has when the jitter is assumed to have a uniform distribution in the interval $-5 \mathrm{~nm}$ to $5 \mathrm{~nm}$, see Fig. 20. The calculation of the theoretical values was carried out in the same way as in Eq. (13) but with a subset $\mathbf{R}_{c q}^{\prime}$ of all quadrupoles and the variance of a uniform rather than

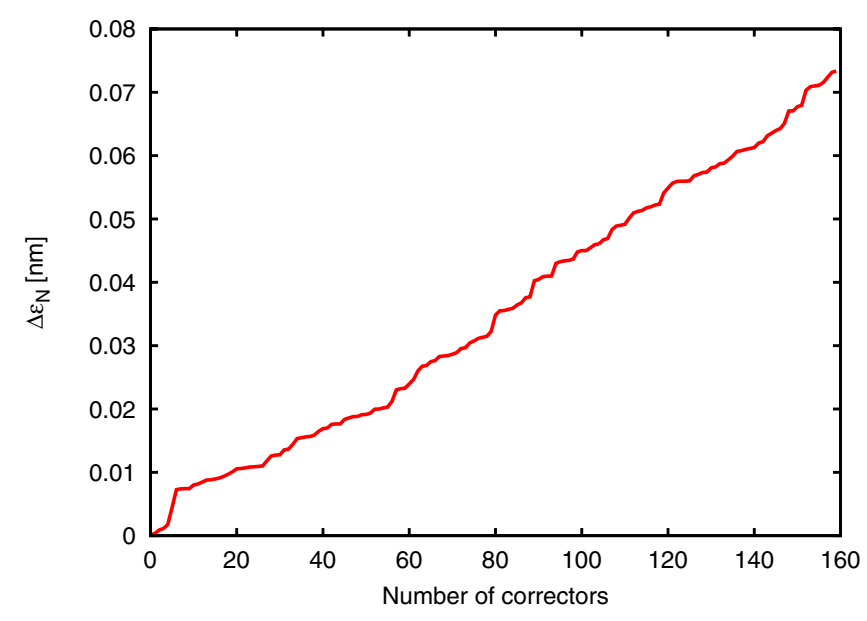

FIG. 20. (Color) Emittance growth caused by a step size error of $10 \mathrm{~nm}$ for a varying number of selected quadrupoles (correctors).
Gaussian distribution. For a step size $s_{q}$, the additional emittance growth is

$$
\left\langle\Delta \epsilon_{N}\right\rangle \approx \frac{s_{q}^{2}}{12}\left\|\mathbf{R}_{c q}^{\prime}\right\|_{F}^{2} .
$$

In this way it was shown that, if all 1324 quadrupoles of the main linac are used, the emittance growth will increase by $\Delta \epsilon_{N} \approx 0.72 \mathrm{~nm}$ for the given step size error. By instead reducing the number of correctors to 60 , the emittance growth caused by the $10 \mathrm{~nm}$ step size is seen to decrease to $\approx 0.023 \mathrm{~nm}$ (in excellent agreement with simulations). The reduction of the number of correctors is, however, done at the cost of a higher emittance growth rate $(\approx 10 \%$ increase $)$ and faster trajectory deviations $(\approx 10 \%)$. Alternatively, the construction of the feedback system can be performed using, preferably, quadrupoles which contribute less to $\left\|\mathbf{R}_{c q}^{\prime}\right\|_{F}^{2}$. These quadrupoles are weaker but also more robust (for step size errors). For a feedback system based on 80 "weak" quadrupoles the emittance growth caused by the $10 \mathrm{~nm}$ step size error was $\approx 0.020 \mathrm{~nm}$ with emittance and trajectory performance as for the 60 correctors mentioned above. Both by using fewer or weaker quadrupoles, larger quadrupole displacements (corrector changes) will be required. This may affect the sensitivity to relative corrector errors. The results of a study of relative corrector errors is shown in Table III.

A feedback system optimized for the optics of the machine and the ground motion spectrum of the site was also seen to very efficiently reduce the effect of certain other imperfections: slow drifts of quadrupoles and of the injected beam. A quadrupole slowly drifting away from the ideal position would be very severe if no feedback is used. Already at a distance of $\approx 21 \mathrm{~nm}$ from the ideal position, the most harmful quadrupole would cause an emittance growth of $0.15 \mathrm{~nm}$. Both the basic feedback system based on 1324 BPMs and the optimized feedback system efficiently reduce the sensitivity, see Fig. 21.

Similarly, if the injected beam slowly drifts from the ideal position, it would quickly cause large emittance growth if no feedback is used. By using a basic feedback system or, even better, an optimized feedback system, the

TABLE III. Additional emittance growth for imperfect compared to ideal correctors. The constant error can be thought of as a corrector strength calibration error which is the same from pulse to pulse. The noise is a corrector strength error varying independently from one pulse to the other. The emittance is stated in $\left[10^{-3} \mathrm{~nm}\right]$.

\begin{tabular}{lcccc}
\hline \hline & \multicolumn{3}{c}{ Constant error } & Noise \\
Feedback setup & $\sigma=1 \%$ & $\sigma=3 \%$ & $\sigma=5 \%$ & $\sigma=1 \%$ \\
\hline 80 correctors & 0.29 & 2.4 & 6.7 & 1.2 \\
60 correctors & 0.53 & 4.2 & 11 & 1.9 \\
80 weak correctors & 0.22 & 3.0 & 9.4 & 0.82 \\
\hline \hline
\end{tabular}




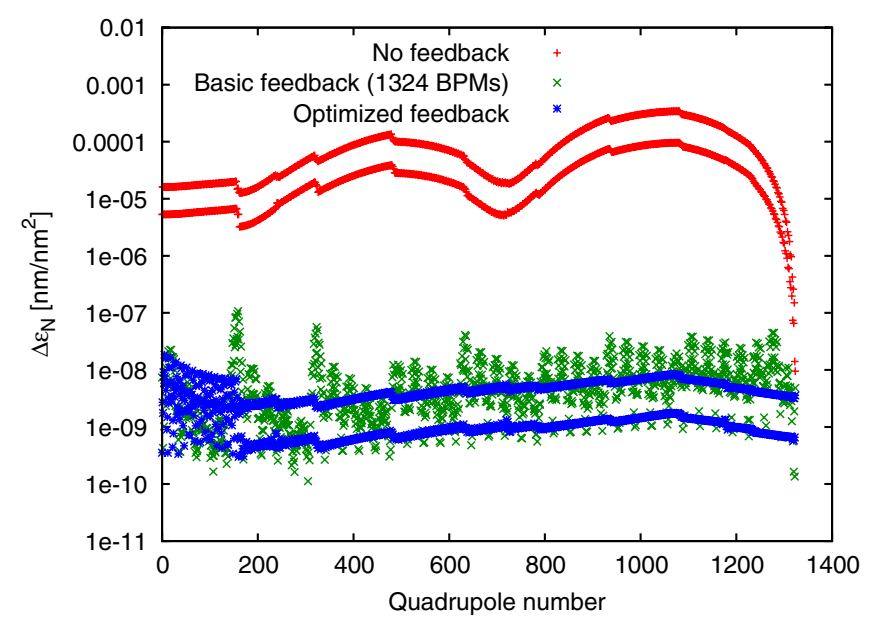

FIG. 21. (Color) Emittance growth caused by a displacement of a quadrupole. Without feedback, even small displacements could have severe consequences, in particular, for certain extra sensitive quadrupoles. By using a basic or optimized feedback system, the tolerance to such displacements is significantly improved.

TABLE IV. Emittance growth caused by a displacement of the injected beam. The effect is severe if no feedback is used but is strongly improved by the use of feedback. The optimized feedback system is particularly efficient.

\begin{tabular}{lc}
\hline \hline \multicolumn{1}{c}{ Feedback setup } & $\Delta \epsilon_{N} / \Delta d^{2}\left[\mathrm{~nm} / \mu \mathrm{m}^{2}\right]$ \\
\hline No feedback & 5.13 \\
Basic feedback (1324 BPMs) & $2.20 \times 10^{-2}$ \\
Optimized feedback & $5.09 \times 10^{-4}$ \\
\hline \hline
\end{tabular}

tolerance to displacements of the injected beam is significantly improved, see Table IV.

\section{B. Optimized multipulse emittance feedbacks}

The feedback system discussed above was optimized to minimize the emittance of single pulses at the end of the machine. This emittance only depends on the distribution of the particles in the pulse. Because of jitter, the final beam position and angle of the pulse as a whole vary quickly from one pulse to the next, leading to a larger distribution for the particles in several pulses, and hence to a larger effective emittance. Since a feedback cannot be used to correct the jitter effects, a more relevant measure for maximization of luminosity may instead be the multipulse emittance, which is defined as the emittance of an ensemble of consecutive pulses. In this way, the relative position and angle of the pulses are also taken into account. In order to determine the multipulse emittance growth caused by beam or element jitter, Eqs. (7)-(9) have to be studied in more detail. Generalization of the second-order emittance expression to include $P$ pulses may be carried out by forming a single multipulse beam by joining the non-normalized coordinates $\tilde{\mathbf{y}}_{i}$ of each pulse $i$ :

$$
\tilde{\mathbf{y}}_{\mathrm{mp}}=\left(\begin{array}{c}
\tilde{\mathbf{y}}_{P-1} \\
\vdots \\
\tilde{\mathbf{y}}_{1} \\
\tilde{\mathbf{y}}_{0}
\end{array}\right) .
$$

In a corresponding manner, the matrix $\mathbf{H}$ of Eq. (7) has to be extended to obtain a correct emittance expression. To do this, first notice that the macroparticle weights have to be changed to sum up to one. The $P \times n$ weights become

$$
\frac{1}{P}\left(\begin{array}{lllllllll}
w_{1} & w_{2} & \cdots & w_{n} & w_{1} & w_{2} & \cdots & w_{n} & \cdots
\end{array}\right) .
$$

This leads to a new multipulse version of the matrix $\mathbf{H}^{\prime}$ :

$$
\mathbf{H}^{\prime}=\frac{1}{P} \mathbf{H}_{+}^{\prime}-\frac{1}{P^{2}} \mathbf{H}_{-}^{\prime},
$$

where $\mathbf{H}_{+}^{\prime}$ and $\mathbf{H}_{-}^{\prime}$ were introduced in Eq. (9). The factors in front of each of these matrices reflect the modification of macroparticle weights and the fact that each nonzero term of $\mathbf{H}_{+}^{\prime}$ contains one weight factor, and that each term of $\mathbf{H}_{-}^{\prime}$ contains two weight factors. The resulting modification of $\mathbf{H}$ is best understood by studying the derivation of the second-order emittance approximation, see [3], and the new multipulse expression becomes

$$
\Delta \epsilon_{N M} \approx \frac{1}{2} \tilde{\mathbf{y}}_{\mathrm{mp}}^{T} \mathbf{H}_{\mathrm{mp}} \tilde{\mathbf{y}}_{\mathrm{mp}}
$$

with

$$
\begin{aligned}
\mathbf{H}_{\mathrm{mp}}= & \frac{1}{P} \mathbf{H}_{\mathrm{mp}+}-\frac{1}{P^{2}} \mathbf{H}_{\mathrm{mp}-} \\
= & \frac{1}{P}\left(\begin{array}{ccc}
\mathbf{H}_{+} & & \\
& \mathbf{H}_{+} & \\
& & \ddots
\end{array}\right)-\frac{1}{P^{2}}\left(\begin{array}{ccc}
\mathbf{H}_{-} & \mathbf{H}_{-} & \cdots \\
\mathbf{H}_{-} & \mathbf{H}_{-} & \cdots \\
\vdots & \vdots & \ddots
\end{array}\right) .
\end{aligned}
$$

The two new matrices $\mathbf{H}_{+}$and $\mathbf{H}_{-}$are defined as

$$
\mathbf{H}_{+}=\gamma_{r}\left(\begin{array}{cc}
\gamma \mathbf{H}_{+}^{\prime} & -\alpha \mathbf{H}_{+}^{\prime} \\
-\alpha \mathbf{H}_{+}^{\prime} & \beta \mathbf{H}_{+}^{\prime}
\end{array}\right)
$$

and

$$
\mathbf{H}_{-}=\gamma_{r}\left(\begin{array}{cc}
\gamma \mathbf{H}_{-}^{\prime} & -\alpha \mathbf{H}_{-}^{\prime} \\
-\alpha \mathbf{H}_{-}^{\prime} & \beta \mathbf{H}_{-}^{\prime}
\end{array}\right)
$$

In analogy with the jitter-induced emittance growth of a single pulse described in Sec. III B, the multipulse emittance growth may be expressed

$$
\begin{aligned}
\left\langle\Delta \epsilon_{N M}\right\rangle & \approx \frac{\sigma^{2}}{2} \operatorname{Tr}\left(\tilde{\mathbf{R}}_{\mathrm{mp}}^{T} \mathbf{H}_{\mathrm{mp}} \tilde{\mathbf{R}}_{\mathrm{mp}}\right) \\
& =\frac{\sigma^{2}}{2}\left(P \frac{1}{P} \operatorname{Tr}\left(\tilde{\mathbf{R}}^{T} \mathbf{H}_{+} \tilde{\mathbf{R}}\right)-P \frac{1}{P^{2}} \operatorname{Tr}\left(\tilde{\mathbf{R}}^{T} \mathbf{H}_{-} \tilde{\mathbf{R}}\right)\right) \\
& =\sigma^{2}\left(f^{+}-\frac{1}{P} f^{-}\right),
\end{aligned}
$$


where

$$
\begin{aligned}
& f^{+}=\frac{1}{2} \operatorname{Tr}\left(\tilde{\mathbf{R}}^{T} \mathbf{H}_{+} \tilde{\mathbf{R}}\right), \\
& f^{-}=\frac{1}{2} \operatorname{Tr}\left(\tilde{\mathbf{R}}^{T} \mathbf{H}_{-} \tilde{\mathbf{R}}\right) .
\end{aligned}
$$

The multipulse version of the response matrix $\tilde{\mathbf{R}}$ is simply

$$
\tilde{\mathbf{R}}_{\mathrm{mp}}=\left(\begin{array}{cccc}
\tilde{\mathbf{R}} & 0 & \cdots & 0 \\
0 & \tilde{\mathbf{R}} & \cdots & 0 \\
\vdots & \vdots & \ddots & \vdots \\
0 & 0 & \cdots & \tilde{\mathbf{R}}
\end{array}\right),
$$

Observe, that in this case the non-normalized response matrices have been used.

Apparently the multipulse emittance growth caused by jitter consists of one constant term and one term which is inversely proportional to the number of pulses $P$. For $P=$ 1 the expression equals the previously obtained singlepulse expression. For a large number of pulses, the expectation value of the multipulse emittance growth is

$$
\left\langle\Delta \epsilon_{N M}\right\rangle \approx \frac{\sigma^{2}}{2} \operatorname{Tr}\left(\tilde{\mathbf{R}}^{T} \mathbf{H}_{+} \tilde{\mathbf{R}}\right)=\sigma^{2} f^{+}
$$

In the same way as for single-pulse emittance, the individual contributions are simply added when several jitter sources are considered.

In Table V, the multipulse scaling factors are summarized for the three jitter effects described in Sec. III B. In particular for quadrupole jitter, the long-term multipulse effect (proportional to $f^{+}$) is significantly stronger than the single-pulse effect (proportional to $e$ ). The total direct multipulse emittance caused by jitter can for the values of Table II be calculated to be $\Delta \epsilon_{N M} \approx 0.37 \mathrm{~nm}(\approx 170 \%$ more than for single-pulse emittance).

Similarly, the effect of ground motion, feedback, imperfect BPMs, and indirect effects of jitter may be derived for multipulse emittance. Since these effects last over a large number of pulses (rather than being uncorrelated from pulse to pulse), the derivations tend to become more tedious. The multipulse coordinate change can as before be denoted $\tilde{\mathbf{y}}_{\mathrm{mp}}$. From Eq. (33) and the discussion above, it is clear that the multipulse coordinate change induced by a feedback system with imperfect BPMs is

TABLE V. Emittance growth scaling factors for quadrupole, accelerating structure, and injected beam jitter, respectively. All values are given in $\mathrm{nm} / \mu \mathrm{m}^{2}$.

\begin{tabular}{lccc}
\hline \hline \multicolumn{1}{c}{ Jitter type } & $f^{+}$ & $f^{-}$ & $e=f^{+}-f^{-}$ \\
\hline Quadrupole & $3.12 \times 10^{5}$ & $2.25 \times 10^{5}$ & $8.69 \times 10^{4}$ \\
Accelerating structure & 0.331 & 0.107 & 0.224 \\
Beam & 5.90 & 0.77 & 5.13 \\
\hline \hline
\end{tabular}

$$
\begin{aligned}
\tilde{\mathbf{y}}_{\mathrm{mp}}= & \left(\begin{array}{c}
0 \\
\vdots \\
\vdots \\
g(1-g)^{0} \tilde{\mathbf{R}}_{c B^{\prime}} \mathbf{b}_{0}^{\prime}
\end{array}\right)+\left(\begin{array}{c}
0 \\
\vdots \\
g(1-g)^{0} \tilde{\mathbf{R}}_{c B^{\prime}} \mathbf{b}_{1}^{\prime} \\
g(1-g)^{1} \tilde{\mathbf{R}}_{c B^{\prime}} \mathbf{b}_{1}^{\prime}
\end{array}\right)+\cdots \\
& +\left(\begin{array}{c}
g(1-g)^{0} \tilde{\mathbf{R}}_{c B^{\prime}} \mathbf{b}_{P-1}^{\prime} \\
\vdots \\
g(1-g)^{(P-2)} \tilde{\mathbf{R}}_{c B^{\prime}} \mathbf{b}_{P-1}^{\prime} \\
g(1-g)^{(P-1)} \tilde{\mathbf{R}}_{c B^{\prime}} \mathbf{b}_{P-1}^{\prime}
\end{array}\right)=\sum_{i=0}^{P-1} \tilde{\mathbf{y}}_{\mathrm{mp}, i}
\end{aligned}
$$

The first term is the coordinate change caused by the most recent BPM error $\mathbf{b}_{0}^{\prime}$ and therefore only affects the most recent pulse (last row of the block vector). The other terms represent the change caused by BPM errors during previous pulses. The last $P$ pulses are taken into account and the last row of $\tilde{\mathbf{y}}_{\mathrm{mp}}$ is therefore seen to equal Eq. (33).

The emittance growth corresponding to this coordinate change is calculated as

$$
\begin{aligned}
\Delta \epsilon_{N M} & \approx \frac{1}{2} \tilde{\mathbf{y}}_{\mathrm{mp}}^{T} \mathbf{H}_{\mathrm{mp}} \tilde{\mathbf{y}}_{\mathrm{mp}} \\
& =\frac{1}{2} \tilde{\mathbf{y}}_{\mathrm{mp}}^{T}\left(\frac{1}{P} \mathbf{H}_{\mathrm{mp}+}-\frac{1}{P^{2}} \mathbf{H}_{\mathrm{mp}}\right) \tilde{\mathbf{y}}_{\mathrm{mp}} .
\end{aligned}
$$

The expectation value of the emittance growth is easily calculated by first noting that the BPM errors $\mathbf{b}_{i}^{\prime}$ are independent stochastic variables and in analogy with previous calculations, mixed terms do not contribute to the emittance growth. Consequently, the expected emittance growth is

$$
\left\langle\Delta \epsilon_{N M}\right\rangle \approx \frac{1}{2} \sum_{i=0}^{P-1}\left(\frac{1}{P}\left\langle\tilde{\mathbf{y}}_{i}^{T} \mathbf{H}_{\mathrm{mp}+} \tilde{\mathbf{y}}_{i}\right\rangle-\frac{1}{P^{2}}\left\langle\tilde{\mathbf{y}}_{i}^{T} \mathbf{H}_{\mathrm{mp}}-\tilde{\mathbf{y}}_{i}\right\rangle\right) .
$$

This sum can be evaluated by using the very simple format of the block matrices $\mathbf{H}_{\mathrm{mp}+}$ and $\mathbf{H}_{\mathrm{mp}-}$, see Eq. (88). After evaluation of a few geometric sums the following result is obtained:

$$
\begin{aligned}
\left\langle\Delta \epsilon_{N M}\right\rangle \approx & \sigma_{\mathrm{BPM}}^{2}\left[\left(\frac{g}{2-g}-\frac{1}{P} \frac{(1-g)^{2}}{(2-g)^{2}}\right) f_{f b}^{+}\right. \\
& \left.-\left(\frac{1}{P}-\frac{2}{P^{2}} \frac{(1-g)}{g}+\frac{1}{P^{2}} \frac{(1-g)^{2}}{g(2-g)}\right) f_{f b}^{-}\right],
\end{aligned}
$$

where $P$ has been assumed to be large, and transient terms have been neglected in the same way as in Eq. (34). The two new scaling factors are

$$
\begin{aligned}
& f_{f b}^{+}=\frac{1}{2} \operatorname{Tr}\left(\tilde{\mathbf{R}}_{c B^{\prime}}^{T} \mathbf{H}_{+} \tilde{\mathbf{R}}_{c B^{\prime}}\right), \\
& f_{f b}^{-}=\frac{1}{2} \operatorname{Tr}\left(\tilde{\mathbf{R}}_{c B^{\prime}}^{T} \mathbf{H}_{-} \tilde{\mathbf{R}}_{c B^{\prime}}\right) .
\end{aligned}
$$


In the limit that $P \rightarrow \infty$, Eq. (99) becomes

$$
\left\langle\Delta \epsilon_{N M}\right\rangle \approx \sigma_{\mathrm{BPM}}^{2} \frac{g}{2-g} f_{f b}^{+},
$$

which is similar to the corresponding single-pulse expression.

Similarly, the indirect emittance growth caused by feedback and jittering quadrupole can be calculated as

$$
\begin{aligned}
\left\langle\Delta \epsilon_{N M}\right\rangle \approx & \sigma_{q}^{2}\left[\left(\frac{g}{2-g}-\frac{1}{P} \frac{(1-g)^{2}}{(2-g)^{2}}\right) f_{f b, q}^{+}\right. \\
& \left.-\left(\frac{1}{P}-\frac{2}{P^{2}} \frac{(1-g)}{g}+\frac{1}{P^{2}} \frac{(1-g)^{2}}{g(2-g)}\right) f_{f b, q}^{-}\right],
\end{aligned}
$$

where

$$
\begin{aligned}
& f_{f b, q}^{+}=\frac{1}{2} \operatorname{Tr}\left(\mathbf{R}_{B^{\prime} q}^{T} \tilde{\mathbf{R}}_{c B^{\prime}}^{T} \mathbf{H}_{+} \tilde{\mathbf{R}}_{c B^{\prime}} \mathbf{R}_{B^{\prime} q}\right), \\
& f_{f b, q}^{-}=\frac{1}{2} \operatorname{Tr}\left(\mathbf{R}_{B^{\prime} q}^{T} \tilde{\mathbf{R}}_{c B^{\prime}}^{T} \mathbf{H}_{-} \tilde{\mathbf{R}}_{c B^{\prime}} \mathbf{R}_{B^{\prime} q}\right) .
\end{aligned}
$$

In the limit that $P \rightarrow \infty$, Eq. (103) becomes

$$
\left\langle\Delta \epsilon_{N M}\right\rangle \approx \sigma_{q}^{2} \frac{g}{2-g} f_{f b, q}^{+} .
$$

The expressions for accelerating structure jitter and injected beam jitter are analogous.

For ground motion and feedback, calculations become slightly more tedious. In the same way as above, the total coordinate change $\tilde{\mathbf{y}}_{\mathrm{mp}}$ after $P$ pulses can be calculated as

$$
\begin{aligned}
\tilde{\mathbf{y}}_{\mathrm{mp}} & =\left(\begin{array}{c}
0 \\
\vdots \\
\vdots \\
\tilde{\mathbf{R}}_{0} \mathbf{r}_{0}
\end{array}\right)+\left(\begin{array}{c}
0 \\
\vdots \\
\tilde{\mathbf{R}}_{0} \mathbf{r}_{1} \\
\tilde{\mathbf{R}}_{1} \mathbf{r}_{1}
\end{array}\right)+\cdots+\left(\begin{array}{c}
\tilde{\mathbf{R}}_{0} \mathbf{r}_{P-1} \\
\vdots \\
\tilde{\mathbf{R}}_{(P-2)} \mathbf{r}_{P-1} \\
\tilde{\mathbf{R}}_{(P-1)} \mathbf{r}_{P-1}
\end{array}\right) \\
& =\sum_{i=0}^{P-1} \tilde{\mathbf{y}}_{\mathrm{mp}, i},
\end{aligned}
$$

where

$$
\begin{aligned}
\tilde{\mathbf{R}}_{i} & =\tilde{\mathbf{R}}_{c g}+\tilde{\mathbf{R}}_{c B^{\prime}} \mathbf{R}_{B^{\prime} g}-(1-g)^{i} \tilde{\mathbf{R}}_{c B^{\prime}} \mathbf{R}_{B^{\prime} g} \\
& =\tilde{\mathbf{R}}_{S}+(1-g)^{i} \tilde{\mathbf{R}}_{D} .
\end{aligned}
$$

The auxiliary matrices $\tilde{\mathbf{R}}_{D}$ and $\tilde{\mathbf{R}}_{S}$ are in this case

$$
\begin{gathered}
\tilde{\mathbf{R}}_{D}=\tilde{\mathbf{R}}_{c B^{\prime}} \mathbf{R}_{B^{\prime} g}, \\
\tilde{\mathbf{R}}_{S}=\tilde{\mathbf{R}}_{c g}+\tilde{\mathbf{R}}_{c B^{\prime}} \mathbf{R}_{B^{\prime} g} .
\end{gathered}
$$

The stochastic variable $\mathbf{r}_{i}$ represents the relative girder displacements at pulse $i$ (as for the single-pulse calculations). The expected emittance growth after $P$ pulses can be calculated in a similar way as before. If transient terms are dropped, the result becomes

$$
\begin{aligned}
\left\langle\Delta \epsilon_{N M}\right\rangle \approx & \sigma_{g}^{2}\left[\frac{P+1}{2} f_{\mathrm{gm}, 0}^{+}-\left(\frac{P}{3}+\frac{1}{2}+\frac{1}{6 P}\right) f_{\mathrm{gm}, 0}^{-}\right. \\
& -\frac{2}{g}\left(1-\frac{1}{P} \frac{1-g}{g}\right) f_{\mathrm{gm}, 1}^{+} \\
& +\frac{2}{g}\left(\frac{1}{2}+\frac{1}{2 P}-\frac{1}{P^{2}} \frac{1-g}{g^{2}}\right) f_{\mathrm{gm}, 1}^{-} \\
& +\frac{1}{g(2-g)}\left(1-\frac{1}{P} \frac{(1-g)^{2}}{g(2-g)}\right) f_{\mathrm{gm}, 2}^{+} \\
& \left.-\frac{1}{g^{2}}\left(\frac{1}{P}-\frac{2}{P^{2}} \frac{1-g}{g}+\frac{1}{P} \frac{(1-g)^{2}}{g(2-g)}\right) f_{\mathrm{gm}, 2}^{-}\right],
\end{aligned}
$$

where the scaling factors are

$$
\begin{aligned}
& f_{\mathrm{gm}, 0}^{ \pm}=\frac{1}{2} \operatorname{Tr}\left(\tilde{\mathbf{R}}_{S}^{T} \mathbf{H}_{ \pm} \tilde{\mathbf{R}}_{S}\right), \\
& f_{\mathrm{gm}, 1}^{ \pm}=\frac{1}{2} \operatorname{Tr}\left(\tilde{\mathbf{R}}_{S}^{T} \mathbf{H}_{ \pm} \tilde{\mathbf{R}}_{D}\right), \\
& f_{\mathrm{gm}, 2}^{ \pm}=\frac{1}{2} \operatorname{Tr}\left(\tilde{\mathbf{R}}_{D}^{T} \mathbf{H}_{ \pm} \tilde{\mathbf{R}}_{D}\right) .
\end{aligned}
$$

Apparently, the multipulse emittance growth rate is

$$
\begin{aligned}
\frac{d\left\langle\Delta \epsilon_{N M}\right\rangle}{d P} \approx & \sigma_{g}^{2}\left(\frac{1}{2} f_{\mathrm{gm}, 0}^{+}-\frac{1}{3} f_{\mathrm{gm}, 0}^{-}\right) \\
= & \sigma_{g}^{2} \operatorname{Tr}\left(\tilde{\mathbf{R}}_{c g}^{T} \mathbf{H}_{*} \tilde{\mathbf{R}}_{c g}+2 \tilde{\mathbf{R}}_{c g}^{T} \mathbf{H}_{*} \tilde{\mathbf{R}}_{c B^{\prime}} \mathbf{R}_{B^{\prime} g}\right. \\
& \left.+\mathbf{R}_{B^{\prime} g}^{T} \tilde{\mathbf{R}}_{c B^{\prime}}^{T} \mathbf{H}_{*} \tilde{\mathbf{R}}_{c B^{\prime}} \mathbf{R}_{B^{\prime} g}\right),
\end{aligned}
$$

where $\mathbf{H}_{*}=(1 / 4) \mathbf{H}_{+}-(1 / 6) \mathbf{H}_{-}$. This is the same expression as obtained in Eq. (51) apart from a different coordinate normalization. The same method as described in Sec. IVA may therefore be used to minimize Eq. (115).

The minimum achievable emittance growth was initially seen to be quite large and by looking at the obtained solution in non-normalized coordinates, it became clear that the existing BPMs could not be used to predict the beam position and angle at the end of the machine accurately enough. By adding two BPMs, separated by a phase advance of $90^{\circ}$, at the end of the linac, this problem was however solved. The number of required BPM patterns could, as in case of single-pulse emittance, be shown to be approximately $25-30$, see Fig. 22 . Once again, the decision was to use 30 BPM patterns. The optimal feedback performance for this number of BPM patterns is a multipulse emittance growth rate of $0.876 \times$ $10^{-4} \mathrm{~nm} /$ minute and a maximum BPM standard deviation of $0.145 \mu \mathrm{m} / \mathrm{min}^{1 / 2}$.

In an analogous manner to the construction described in Sec. IVA, Eq. (65) had to be solved to find the desired linear combinations of dipole correctors. By choosing 80 good correctors in the same way as before, the optimal feedbacks could be constructed with reasonable accuracy. The multipulse emittance growth rate was determined theoretically to be $2.31 \times 10^{-4} \mathrm{~nm} / \mathrm{min}$ and the maxi- 


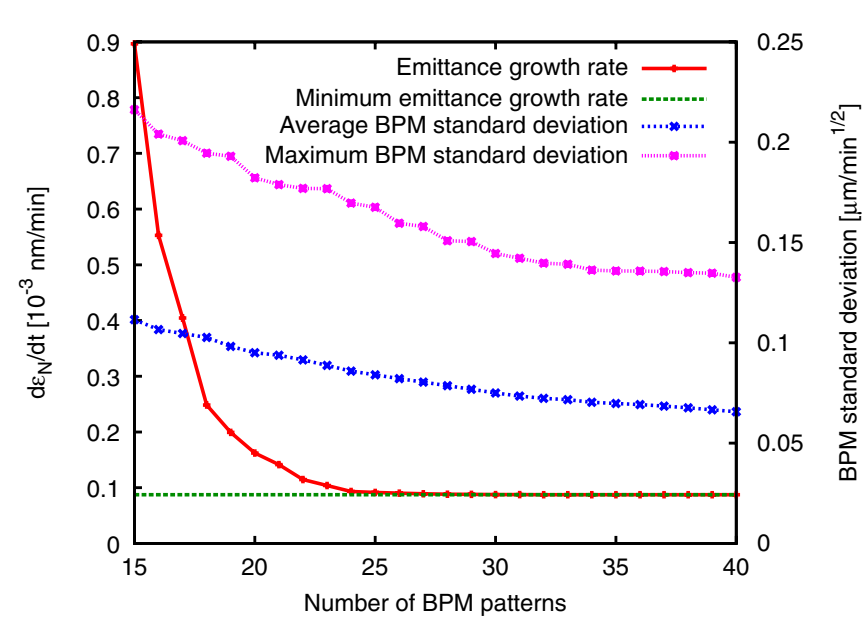

FIG. 22. (Color) Minimum achievable multipulse emittance growth rate for different numbers of BPM patterns. The 25-30 most important patterns have to be considered to achieve optimal performance. The figure also shows how well the trajectory can be controlled with information from a limited number of BPM patterns.

mum BPM standard deviation to be $0.702 \mu \mathrm{m} / \mathrm{min}^{1 / 2}$ (see Fig. 23). The sensitivity to imperfect system knowledge was seen to be acceptable for $0.01 \%$ and $0.1 \%$ errors (cf. Sec. IVA 1).

When the growth rate performance had been optimized, the constant emittance growth terms, caused by imperfect feedback BPMs and the indirect effect of feedback and jitter, had to be minimized. In order to do this, the gain had to be minimized in the same way as in Sec. IVA 2. The optimal value of a single gain parameter was seen to be 0.070 , leading to a constant multipulse emittance growth term of $0.148 \mathrm{~nm}$. Even though optimization of multiple gain parameters, each corresponding to a BPM pattern, was

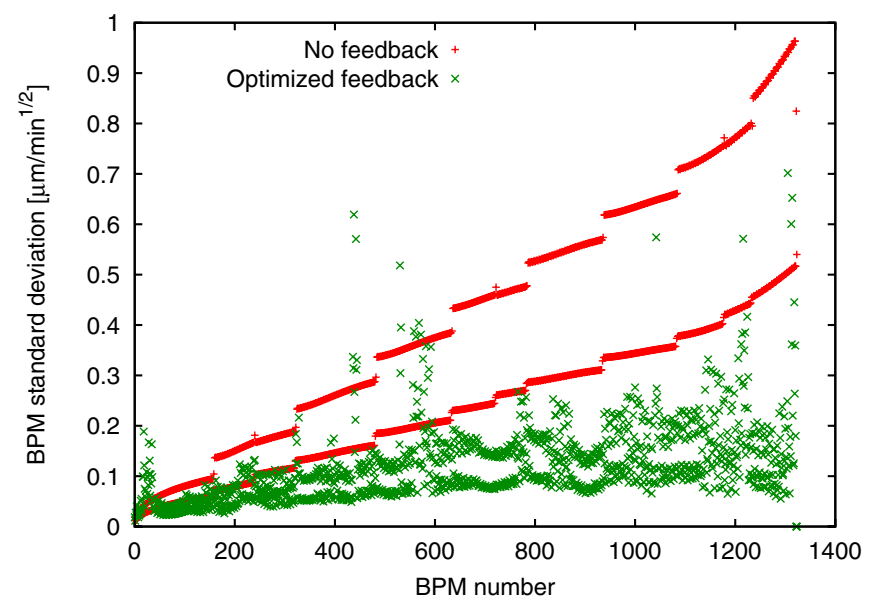

FIG. 23. (Color) Theoretical standard deviation of BPM readings for a large number of seeds. The maximum deviation for the optimized feedback is seen to be $\approx 0.7 \mathrm{~nm}$, just as for the feedback system optimized for single-pulse emittance.

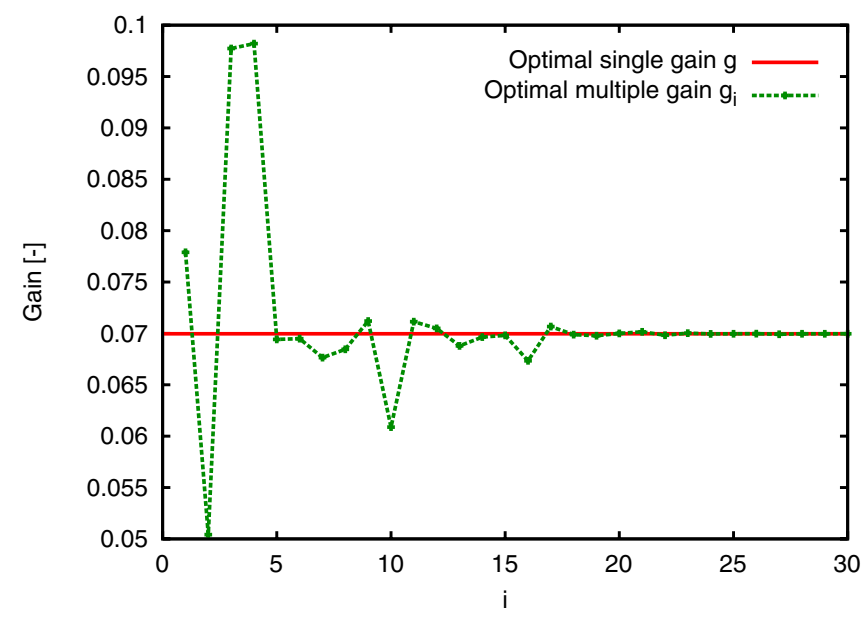

FIG. 24. (Color) Optimal values of a single gain parameter and of multiple gain parameters (for each BPM pattern $i$ ). The optimal single gain $g \approx 0.07$ was used as a starting point for the multiple gain parameters $g_{i}$ during the optimization procedure.

seen to offer only a little additional reduction of the emittance growth in case of single-pulse emittance, it was tested in this case as well. Once again, the achievable reduction was small, giving an emittance growth of $0.144 \mathrm{~nm}$, see Figs. 24 and 25. Apparently, this constant feedback-induced term is much larger than in case of single-pulse emittance (it is however small compared to the direct emittance growth of $0.371 \mathrm{~nm}$ caused by jitter). The reason is most likely that the feedback correction of the beam position and angle, which are both very important for the multipulse emittance, relies heavily on the information obtained from the two new BPMs added at the end

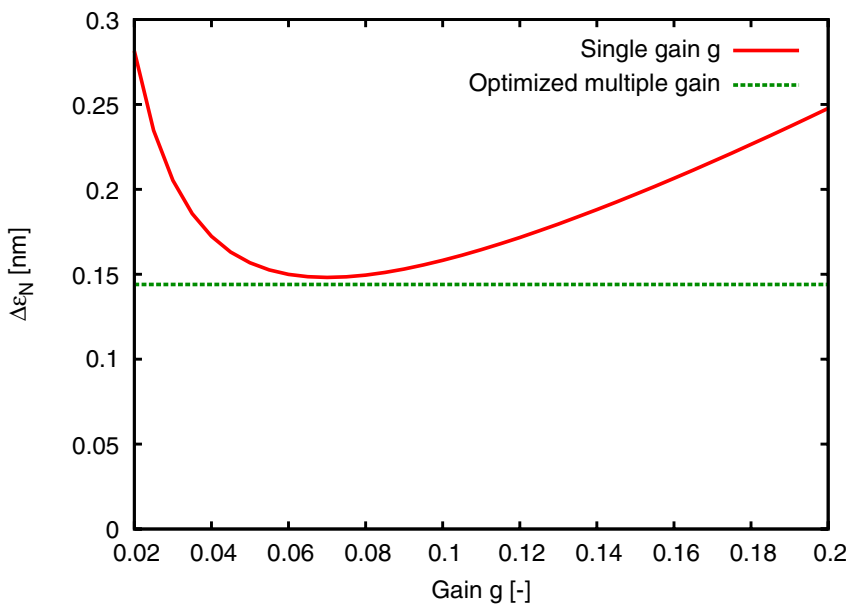

FIG. 25. (Color) Total gain-dependent multipulse emittance growth for various values of the single gain parameters and for the optimal multiple gains (see Fig. 24). For the optimal single gain $g \approx 0.07$, the multipulse emittance growth is $\Delta \epsilon_{N M} \approx$ $0.148 \mathrm{~nm}$ compared to $\Delta \epsilon_{N M} \approx 0.144 \mathrm{~nm}$ for optimized multiple gain parameters. 
TABLE VI. Contributions of the different imperfections to the total gain-dependent multipulse emittance growth.

\begin{tabular}{lc}
\hline \hline \multicolumn{1}{c}{ Source } & $\Delta \epsilon_{N M}[\mathrm{~nm}]$ \\
\hline Slow feedback (nonunit gain) & 0.076 \\
Imperfect BPMs & 0.054 \\
Feedback and quadrupole jitter & 0.012 \\
Feedback and accelerating structure jitter & Negligible \\
Feedback and injected beam jitter & 0.002 \\
\hline \hline
\end{tabular}

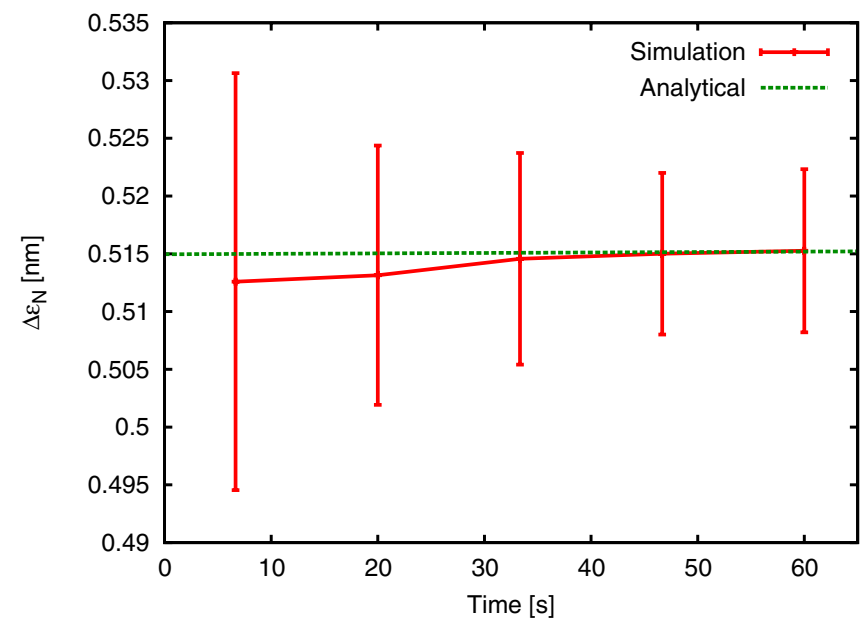

FIG. 26. (Color) Multipulse emittance growth caused by imperfections according to Table II. The result of simulations was obtained as an average of 50 random seeds and agrees very well with analytical predictions.

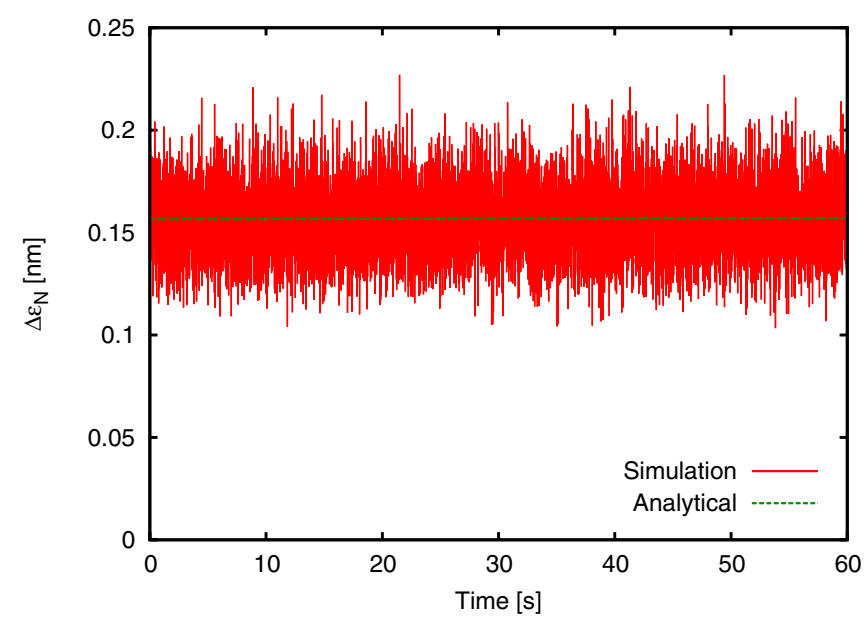

FIG. 27. (Color) Single-pulse emittance growth along the CLIC main linac during 1 min of operation when multipulse optimized feedbacks are used. The result is nearly indistinguishable from what was obtained with feedbacks optimized for single-pulse emittance cf. Figure 19. of the machine. These BPMs were simply assumed to have the same resolution $\left(\sigma_{\mathrm{BPM}}=0.1 \mu \mathrm{m}\right)$ as all the other ones even though BPMs with an even better precision would be desirable here. The contributions of different sources to the multipulse emittance growth of $0.144 \mathrm{~nm}$ are shown in Table VI.

A complete simulation of 50 machines showed that the multipulse emittance performance is as expected, see Fig. 26. The single-pulse emittance performance of the feedback system optimized for multipulse emittance was seen to be nearly identical to that of the feedbacks constructed in Sec. IVA, see Figs. 19 and 27.

\section{CONCLUSIONS}

An efficient way of simulating dynamic imperfections caused by misalignments of large number of elements has been suggested. The response matrix formalism so introduced was also used to find analytical expressions for emittance growth caused by jitter of beam line elements and of the injected beam. In addition, emittance growth due to ground motion, feedback, and imperfect BPMs has been determined analytically. Expressions valid for emittance of single pulses as well as of ensembles of pulses (so-called multipulse emittance) have been derived.

The analytical expressions were used to determine the total emittance growth due to jitter in case of the CLIC main linac: $0.138 \mathrm{~nm}$ (single-pulse), $0.371 \mathrm{~nm}$ (multipulse). The formalism was also used to design a feedback system optimized for emittance control. By adapting the feedback system to the optics of the CLIC main linac and the ground motion spectrum of the site, and in addition selecting carefully 80 useful dipole correctors, its performance was seen to be approximately 10 times better (in terms of emittance growth rate) than that of traditional trajectory feedbacks.

The single-pulse emittance growth rate of the optimized feedback was determined to be approximately $2-3 \times$ $10^{-4} \mathrm{~nm} / \mathrm{min}$ (theoretically as low as $1.7 \times$ $\left.10^{-4} \mathrm{~nm} / \mathrm{min}\right)$. The trajectory control was at the same time seen to be acceptable and the system could ideally counteract ground motion for up to 14 hours before further alignment is required. The gain was optimized such that the feedback system itself induce an emittance growth of only $0.018 \mathrm{~nm}$. Two ways of optimizing the gain were tested: optimization of a single gain parameter, and optimization of individual gain parameters corresponding to different directions in a transformed space of BPM readings. While the second method is more general, it was seen to improve emittance only marginally.

For a feedback system optimized for multipulse emittance, the multipulse emittance growth was $\approx 2.3 \times$ $10^{-4} \mathrm{~nm} / \mathrm{min}$. The trajectory control was seen to be good. By optimizing the gain parameters of the feedback system, a feedback-induced multipulse emittance growth of $0.144 \mathrm{~nm}$ was obtained. 
The optimized feedbacks, which rely on knowledge of the optics of the machine, were tested to guarantee that requirements on system knowledge are not too demanding. It was seen that, while being more sensitive than traditional feedbacks, the optimized feedbacks will work well even for $0.1 \%$ quadrupole strength errors along the machine.

The effect of dipole corrector imperfections have also been tested with promising results. The most severe effect may be that of step size errors. By simulating the dipole correctors as displacements of quadrupoles, it was seen that a minimum step size of $10 \mathrm{~nm}$ gives rise to an emittance growth of approximately $0.020-0.037 \mathrm{~nm}$ depending on the exact design. For comparison, a feedback system using all 1324 dipole correctors would cause an emittance increase of roughly $0.72 \mathrm{~nm}$.

The optimized feedback system was in addition seen to very efficiently reduce the effect of individual quadrupoles drifting away from the ideal position. Similarly the effect of a drifting injected beam was seen to be significantly suppressed.

\section{ACKNOWLEDGMENTS}

I would like to thank Daniel Schulte for reviewing the paper and for useful discussions about the wide range of imperfections affecting feedback performance. This work is supported by the Commission of the European Communities under the 6th Framework Programme "Structuring the European Research Area," Contract No. RIDS-011899.

[1] R. W. Assmann et al., Report No. CERN-2000-008, 2000.
[2] D. Schulte, Report No. CERN-OPEN-2003-015, 2003.

[3] P. Eliasson and D. Schulte, Phys. Rev. ST Accel. Beams 11, 011002 (2008).

[4] N. Leros and D. Schulte, Proceedings of the Particle Accelerator Conference, Chicago, IL, 2001 (IEEE, New York, 2001), p. 1687.

[5] N. Leros and D. Schulte, Proceedings of the 8th European Particle Accelerator Conference, Paris, 2002 (EPS-IGA and CERN, Geneva, 2002), p. 464.

[6] B. A. Baklakov et al., Proceedings of IEEE Particle Accelerator Conference, San Francisco, California, 1991 (IEEE, New York, 1991), p. 3273.

[7] D. Schulte, Proceedings of the European Particle Accelerator Conference, Stockholm, 1998 (IOP, London, 1998), p. 478.

[8] E. T. D'Amico, G. Guignard, N. Leros, and D. Schulte, Proceedings of the Particle Accelerator Conference, Chicago, IL, 2001, Ref. [4], p. 3033.

[9] S. Redaelli, R. W. Assmann, W. Coosemans, and W. Schnell, Proceedings of the 8th European Particle Accelerator Conference, Paris, 2002 (EPS-IGA and CERN, Geneva, 2002), p. 485.

[10] W. Schnell, Report No. CERN-OPEN-2001-001, 2001.

[11] G. H. Golub and C.F. Van Loan, Matrix Computations (The Johns Hopkins University Press, Baltimore, Maryland, 1996), 3rd ed.

[12] L. Hendrickson, N. Phinney, and P. Raimondi, Proceedings of the 20th International Linac Conference, Monterey, CA, 2000 (SLAC, Stanford, 2000), p. 515.

[13] L. Hendrickson et al., Proceedings of the 20th International Linac Conference, Monterey, CA, 2000, Ref. [12], p. 74.

[14] A. Sery and O. Napoly, Phys. Rev. E 53, 5323 (1996).

[15] R. Assmann, C. Salsberg, and C. Montag, Proceedings of 18th International Linac Conference, Geneva, Switzerland, 1996 (CERN, Geneva, 1996), p. 482. 